\title{
Approximate Integration Formulas For Certain Spherically Symmetric Regions
}

\author{
By A. H. Stroud and Don Secrest
}

1. Introduction. In this paper we present approximate integration formulas for the integrals

$$
\begin{aligned}
& U=\int_{-\infty}^{\infty} \cdots \int_{-\infty}^{\infty} \exp \left[-x_{1}{ }^{2}-\cdots-x_{n}{ }^{2}\right] f\left(x_{1}, \cdots, x_{n}\right) d x_{n} \cdots d x \\
& V=\int_{-\infty}^{\infty} \cdots \int_{-\infty}^{\infty} \exp \left[-\sqrt{{x_{1}}^{2}+\cdots+x_{n}}\right] f\left(x_{1}, \cdots, x_{n}\right) d x_{n} \cdots d x_{1}
\end{aligned}
$$

for $n \geqq 2$. The formulas have the form

$$
\int \cdots_{R} \int w\left(x_{1}, \cdots, x_{n}\right) f\left(x_{1}, \cdots, x_{n}\right) d x \simeq \sum_{i=1}^{N} A_{i} f\left(\nu_{i 1}, \cdots, \nu_{i n}\right)
$$

where the $A_{i}$ are constants and the $\nu_{i}=\left(\nu_{i 1}, \cdots, \nu_{i n}\right)$ are points in the space. Each formula is exact for all polynomials up to and including a specified degree $k$ where, as usual, $k$ is called the degree of the formula.

In Section 2 we give formulas of degrees 2, 3, 5 and 7 which are similar to previously developed formulas for other regions. The formulas of degree 2 are discussed by Stroud [14] for an arbitrary region; the formulas of degrees 3, 5 and 7 are similar to formulas for spheres given by Hammer and Stroud [4] and Ditkin [3].

In Sections 3, 4 and 5 we develop spherical product type formulas of arbitrarily high degree for $U$ and $V$. These formulas have degree $2 h-1(h=1,2, \cdots)$ and use $h^{n}$ points for even $h$ and $h^{n}-h^{n-1}+1$ points for odd $h$. These formulas are obtained by products of one-dimensional formulas and are similar to formulas for the circle and 3 -sphere given by Peirce $[10,11]$ and recently extended to the $n$ sphere by Hetherington [6]. The spherical product formulas are most useful in 2 and 3 dimensions since in higher dimensions the numbers of points in the formulas become very large. In Section 4 we tabulate one-dimensional formulas, which are particular to the integrals $U$ and $V$, from which product formulas for $n=2,3,4$ may be constructed.

From the formulas we give for $U$ and $V$ we can obtain formulas for any integrals of the form

$$
\int_{-\infty}^{\infty} \cdots \int_{-\infty}^{\infty} e^{-\varphi(y)} f\left(y_{1}, \cdots, y_{n}\right) d y, \int_{-\infty}^{\infty} \cdots \int_{-\infty}^{\infty} e^{-\sqrt{\varphi(y)}} f\left(y_{1}, \cdots, y_{n}\right) d y
$$

where

$$
\begin{aligned}
\varphi(y)=a_{11} y_{1}{ }^{2}+\cdots+a_{n n} y_{n}{ }^{2}+2 a_{12} y_{1} y_{2}+\cdots+2 a_{n-1, n} y_{n-1} y_{n} & \\
& +a_{01} y_{1}+\cdots+a_{0 n} y+a_{00}
\end{aligned}
$$

Received January 10, 1962. The work presented in this paper was supported in part by the United States Air Force, monitored by Aeronautical Research Laboratory, Wright Air Development Center. 
is obtained by a non-singular linear transformation

$$
y=A x
$$

from $x_{1}{ }^{2}+\cdots+x_{n}{ }^{2}$. By the results of Hammer and Wymore [.; ] it follows that transformation (1.3) applied to a formula for $U$ or $V$ will give a formula of the same degree for the corresponding integral (1.2).

The relationship between approximate integration formulas for functions of one variable and orthogonal polynomials in one variable is well known (see, for example, Krylov [9] or Hildebrand [7]). Almost nothing is known about the corresponding relationship in more than one variable. The only formulas which have been obtained from this point of view are the formulas of degree $\tilde{s}$ for planar regions derived by Radon [13]. In Section 6 we make some remarks about what is known concerning this relationship between integration formulas and orthogonal polynomials in $n$ variables. In Section 7 we show that for Cartesian product regions integration formulas of degree $2 k-1$ cannot be obtained from certain types of systems of orthogonal polynomials of degree $k$. In Section 8 we show how the spherical product formulas are related to systems of orthogonal polynomials.

2. Special Formulas of Low Degree. In Tables 1 and 2 we tabulate formulas of degrees 2, 3, 5 and 7 for the integrals $U$ and $V$. We have tabulated only the necessary parameters in the following formulas, the first four of which are for arbitrary $n$; in all of these formulas the indicated points may be rotated about the origin in any manner:

I. A formula of degree 2 for $n$ dimensions using $n+1$ points. The points are vertices of a regular $n$-simplex with centroid at the origin; the coefficients $A_{k}(k=$ $1,2, \cdots, n+1)$ are all equal. We can take the points to be:

$$
\begin{aligned}
\nu_{1} & =(\rho \sqrt{n}, 0,0, \cdots, 0) \\
\nu_{2} & =\left(-\rho \sqrt{\frac{1}{n}}, \rho \sqrt{\frac{(n+1)(n-1)}{n}}, 0, \cdots, 0\right) \\
\nu_{3} & =\left(-\rho \sqrt{\frac{1}{n}},-\rho \sqrt{\frac{n+1}{n(n-1)}}, \rho \sqrt{\frac{(n+1)(n-2)}{n-1}}, \cdots, 0\right) \\
\nu_{n} & =\left(-\rho \sqrt{\frac{1}{n}},-\rho \sqrt{\frac{n+1}{n(n-1)}},-\rho \sqrt{\frac{n+1}{(n-1)(n-2)}}, \cdots, \rho \sqrt{\frac{n+1}{2}}\right) \\
\nu_{n+1} & =\left(-\rho \sqrt{\frac{1}{n}},-\rho \sqrt{\frac{n+1}{n(n-1)}},-\rho \sqrt{\frac{n+1}{(n-1)(n-2)}}, \cdots,-\rho \sqrt{\frac{n+1}{2}}\right) .
\end{aligned}
$$

The tables give the value of $\rho$ and the common coefficient $A$.

II. A formula of degree 3 for $n$ dimensions using $2 n$ points. We use the points

$$
\begin{gathered}
\nu_{1}=-\nu_{n+1}=(\nu, 0, \cdots, 0) \\
\nu_{2}=-\nu_{n+2}=(0, \nu, \cdots, 0) \\
\cdots \\
\nu_{n}=-\nu_{2 n}=(0,0, \cdots, \nu)
\end{gathered}
$$

with equal coefficient $A$. 


\section{Table 1}

Formulas for $\int_{-\infty}^{\infty} \cdots \int_{-\infty}^{\infty} \exp \left(-x_{1}{ }^{2}-\cdots-x_{n}{ }^{2}\right) f\left(x_{1}, \cdots, x_{n}\right) d x_{1} \cdots d x_{n}$

I. Degree $2, n+1$ points.

$$
\rho=\frac{\sqrt{2}}{2} \quad A=\frac{1}{n+1} \mathrm{~V}
$$

II. Degree $3,2 n$ points.

$$
\nu=\sqrt{\frac{n}{2}} \quad A=\frac{1}{2 n} \mathrm{~V}
$$

III. Degree $3,2^{n}$ points.

$$
\nu=\frac{\sqrt{2}}{2} \quad A=\frac{1}{2^{n}} \mathrm{~V}
$$

IV. Degree $5,2 n^{2}+1$ points.

$$
\begin{aligned}
& A=\frac{2}{n+2} \mathrm{~V} \\
& \nu=\sqrt{\frac{n+2}{2}} \quad B=\frac{4-n}{2(n+2)^{2}} \mathrm{~V} \\
& \xi=\sqrt{\frac{n+2}{4}} \quad C=\frac{1}{(n+2)^{2}} \mathrm{~V} \\
& \text { V. Degree } 5, n=2,7 \text { points. } \\
& \nu=\sqrt{2}=1.41421356237 \\
& A=\frac{1}{2} \mathrm{~V}=1.57079632679 \\
& \xi=\frac{\sqrt{2}}{2}=0.707106781187 \\
& B=\frac{1}{12} \mathrm{~V}=0.261799387799 \\
& \eta=\frac{\sqrt{6}}{2}=1.22474487139
\end{aligned}
$$

VI. Degree 7, $n=2,12$ points.

$\nu=\sqrt{3}=1.73205080757$

$\xi=\sqrt{\frac{9-3 \sqrt{5}}{8}}=0.535233134660$

$\eta=\sqrt{\frac{9+3 \sqrt{5}}{8}}=1.40125853844$

$$
\begin{aligned}
& A=\frac{1}{36} \mathrm{~V}=0.0872664625997 \\
& B=\frac{5+2 \sqrt{5}}{45} \mathrm{~V}=0.661279838445 \\
& C=\frac{5-2 \sqrt{5}}{45} \mathrm{~V}=0.0368518623526
\end{aligned}
$$

VII. Degree $5, n=3,13$ points.

$\nu=\sqrt{\frac{5-\sqrt{5}}{4}}=0.831253875555$

$\xi=\sqrt{\frac{5+\sqrt{5}}{4}}=1.34499702393$

$$
\begin{aligned}
& A=\frac{2}{5} \mathrm{~V}=2.22733119873 \\
& B=\frac{1}{20} \mathrm{~V}=0.278416399841
\end{aligned}
$$

VIII. Degree $5, n=3,15$ points.

$\nu=\sqrt{\frac{5}{2}}=1.58113883008$

$$
\begin{aligned}
& A=\frac{2}{5} \mathrm{~V}=\underset{(0.0000000000)}{2.22733119873} \\
& B=\frac{1}{25} \mathrm{~V}=0.222733119873
\end{aligned}
$$


VIII. Degree $5, n=3,15$ points-Cont.

$$
\begin{aligned}
& \left(\sqrt{\frac{5}{4}}=1.11803398875\right) \\
\eta= & \sqrt{\frac{5}{6}}=0.912870929175 \\
& \left(\sqrt{\frac{5}{2}}=1.58113883008\right)
\end{aligned}
$$

$$
\begin{aligned}
\left(\frac{4}{25} \mathrm{~V}=0.890932479491\right) \\
C=\frac{9}{200} \mathrm{~V}=0.250574759857 \\
\left(\frac{1}{200} \mathrm{~V}=0.0278416399841\right)
\end{aligned}
$$

IX. Degree $5, n=3,21$ points.

$$
\begin{array}{rlrl}
\eta & =\sqrt{\frac{5}{6}=0.912870929175} & A=\frac{2}{5} \mathrm{~V}=2.22733119873 \\
\xi & =\sqrt{\frac{15-5 \sqrt{5}}{12}}=0.564185261572 & B=\frac{3}{100} \mathrm{~V}=0.167049839905 \\
\nu & =\sqrt{\frac{15+5 \sqrt{5}}{12}}=1.47705619075 &
\end{array}
$$

X. Degree $7, n=3,27$ points.

$$
\begin{aligned}
& A=\frac{720 \pm 8 \sqrt{15}}{2205} \mathrm{v}=\frac{1.89647369184}{(1.73998540813)} \\
& \nu=\sqrt{\frac{15 \pm \sqrt{\overline{15}}}{4}}=2.17215235114 \\
& B=\frac{270 \mp 46 \sqrt{15}}{15435} \mathrm{~V}=\underset{(0.161677128128)}{0.0331331807994} \\
& \xi=\sqrt{\frac{6 \mp \sqrt{15}}{2}}=\frac{1.03126540080}{(2.22182170147)} \\
& C=\frac{162 \pm 41 \sqrt{15}}{6174} \mathrm{v}=\underset{(0.0028930077698}{0.28932455621} \\
& \eta=\sqrt{\frac{9 \pm 2 \sqrt{15}}{2}}=\underset{(0.791843831694)}{2.89361078001} \\
& D=\frac{783 \mp 202 \sqrt{15}}{24696} \mathrm{~V}=\underset{(0.352945465837)}{0.000148219093512}
\end{aligned}
$$

XI. Degree $7, n=3,33$ points.

$$
\begin{aligned}
& \nu=\sqrt{\frac{25 \pm 15 \sqrt{2}-5 \sqrt{5} \mp 3 \sqrt{10}}{4}} \\
& A=\frac{80 \pm 8 \sqrt{2}}{245} \mathrm{~V}=\underset{(1.56109306108)}{2.07536603890} \\
& =2.52715405979 \\
& \xi=\sqrt{\frac{25 \pm 15 \sqrt{2}+5 \sqrt{5} \pm 3 \sqrt{10}}{4}} \\
& =4.08902116354 \\
& \text { (1.17050239976) } \\
& \eta=\sqrt{\frac{3 \mp \sqrt{2}}{2}}=\begin{array}{r}
0.890445517038 \\
(1.48563346125)
\end{array} \\
& B=\frac{395 \mp 279 \sqrt{2}}{13720} \mathrm{~V}=\underset{(0.320448990246)}{0.000176309862992} \\
& C=\frac{45 \pm 29 \sqrt{2}}{2744} \mathrm{v}=\underset{(0.00809235264011}{0.17454231979} \\
& \mu=\sqrt{\frac{9 \mp 3 \sqrt{2}+3 \sqrt{5} \mp \sqrt{10}}{4}} \\
& =1.44077111170 \\
& \lambda=\sqrt{\frac{9 \mp 3 \sqrt{2}-3 \sqrt{5} \pm \sqrt{10}}{4}} \\
& \text { (2.40380543513) } \\
& =\begin{array}{r}
0.550325594660 \\
(0.918171973877)
\end{array}
\end{aligned}
$$


TABLE 2 .

Formulas for $\int_{-\infty}^{\infty} \cdots \int_{-\infty}^{\infty} \exp \left(-\sqrt{x_{1}{ }^{2}+\cdots+x_{n}}\right) f\left(x_{1}, \cdots, x_{n}\right) d x_{1} \cdots d x_{n}$.

I. Degree $2, n+1$ points.

$$
\rho=\sqrt{n+1} \quad A=\frac{1}{n+1} \mathrm{~V}
$$

II. Degree 3, $2 n$ points.

$$
\nu=\sqrt{n(n+1)} \quad A=\frac{1}{2 n} \mathbf{V}
$$

III. Degree $3,2^{n}$ points.

$$
\begin{aligned}
& \nu=\sqrt{n+1} \quad A=\frac{1}{2^{n}} \mathrm{~V} \\
& A=\frac{2(2 n+3)}{(n+2)(n+3)} \mathbf{V} \\
& \nu=\sqrt{(n+2)(n+3)} \quad B=\frac{(4-n)(n+1)}{2(n+2)^{2}(n+3)} \mathbf{V} \\
& \xi=\sqrt{\frac{(n+2)(n+3)}{2}} \quad C=\frac{n+1}{(n+2)^{2}(n+3)} \mathbf{V} \\
& \nu=\varsigma \sqrt{5}=4.47213595500 \quad A=\frac{7}{10} \mathrm{~V}=4.39822971502 \\
& \xi=\sqrt{5}=2.23606797750 \quad B=\frac{1}{20} \mathrm{~V}=0.314159265358 \\
& \eta=\sqrt{1} \overline{5}=3.87298334621
\end{aligned}
$$

VI. Degree 7, $n=2,12$ points.

$$
\begin{aligned}
& \nu=\sqrt{42}=6.48074069841 \\
& A=\frac{5}{588} \mathrm{~V}=0.0534284464896 \\
& \xi=\sqrt{\frac{6615-21 \sqrt{74255}}{454}}=1.40212878221 \quad B=\frac{5272105+18733 \sqrt{74255}}{43661940} \mathrm{~V} \\
& \eta=\sqrt{\frac{6615+21 \sqrt{74255}}{454}}=5.21296499519 \quad C=\frac{5272105-18733 \sqrt{74255}}{43661940} \mathrm{~V} \\
& \text { VII. Degree } 5, n=3,13 \text { points. } \\
& \nu=\sqrt{15-3 \sqrt{5}}=2.87954789290 \\
& A=\frac{3}{5} \mathrm{~V}=15.0796447372 \\
& \xi=\sqrt{15+3 \sqrt{5}}=4.65920636294 \quad B=\frac{1}{30} \mathrm{~V}=0.837758040956 \\
& \text { VIII. Degree } 5, n=3,15 \text { points. } \\
& \nu=\sqrt{30}=5.47722557505 \\
& A=\frac{3}{5} \mathrm{~V}=15.0796447372 \\
& \eta=\sqrt{10}=3.16227766017 \\
& B=\frac{2}{75} \mathrm{~V}=0.670206432765 \\
& C=\frac{3}{100} \mathrm{~V}=0.753982236861
\end{aligned}
$$


IX. Degree $5, n=3,21$ points.

$$
\begin{array}{rlrl}
\eta & =\sqrt{10}=3.16227766017 & \\
\xi & =\sqrt{15-5 \sqrt{5}}=1.95439507585 & A & =\frac{3}{5} \mathrm{~V}=15.0796447372 \\
\nu & =\sqrt{15+5 \sqrt{5}}=5.11667273602 & B & =\frac{1}{50} \mathrm{~V}=0.502654824574
\end{array}
$$

$\mathrm{X}$. Degree 7, $n=3,27$ points.

$$
\begin{array}{rlrl}
\nu & =\sqrt{\frac{720-24 \sqrt{130}}{11}=6.37008561364} & A & =\frac{5175-13 \sqrt{130}}{8820} \mathrm{~V}=14.3238878061 \\
\xi & =\sqrt{288+24 \sqrt{130}}=23.6989894726 & B & =\frac{3870+283 \sqrt{130}}{493920} \mathrm{~V}=0.361109968280 \\
\eta & =\sqrt{\frac{-216+24 \sqrt{130}}{7}}=2.86959683639 & C & =\frac{3204-281 \sqrt{130}}{197568} \mathrm{~V} \\
& D & =\frac{4239+373 \sqrt{130}}{197568} \mathrm{~V}=0.0000136185893490
\end{array}
$$

XI. Degree $7, n=3,33$ points.

$$
\begin{aligned}
& \nu=\sqrt{-50+10 \sqrt{5}+10 \sqrt{39}-2 \sqrt{195}} \\
& =2.62339087279 \\
& \begin{array}{r}
\xi=\sqrt{-50-10 \sqrt{5}+10 \sqrt{39}+2 \sqrt{195}} \\
=4.24473559795
\end{array} \\
& \eta=\sqrt{36+4 \sqrt{39}}=7.80896868950 \\
& \mu=\sqrt{54+18 \sqrt{5}+6 \sqrt{39}+2 \sqrt{195}} \\
& =12.6351767567 \\
& \lambda=\sqrt{54-18 \sqrt{5}+6 \sqrt{39}-2 \sqrt{\overline{195}}} \\
& =4.82620806719
\end{aligned}
$$$$
A=\frac{1725-26 \sqrt{39}}{2940} \mathrm{~V}=13.3582233790
$$$$
B=\frac{1065+171 \sqrt{3 \overline{9}}}{54880} \mathrm{~V}=0.976776412186
$$

$$
C=\frac{297-47 \sqrt{39}}{32928} \mathrm{v}=0.00266004517589
$$

III. A formula of degree 3 for $n$ dimensions using the $2^{n}$ points:

$$
( \pm \nu, \pm \nu, \cdots, \pm \nu)
$$

each point having coefficient $A$.

IV. A formula of degree 5 for $n$ dimensions using $2 n^{2}+1$ points. We use the points:

$$
\begin{array}{ll}
(0,0,0, \cdots, 0) \text { coeff. } A \\
( \pm \nu, 0,0, \cdots, 0) & B \\
( \pm \xi, \pm \xi, 0, \cdots, 0) & C
\end{array}
$$

and all points obtained from these by all possible permutations of coordinates; points in the same set are taken with equal coefficients.

The following are specific formulas for 2 and 3 dimensions:

V. A formula of degree 5 for $n=2$ using 7 points:

$$
(0,0) A \quad( \pm \nu, 0) B \quad( \pm \xi, \pm \eta) B .
$$


VI. A formula of degree 7 for $n=2$ using 12 points:

$$
\begin{array}{ll}
( \pm \nu, 0) A & ( \pm \xi, \pm \xi) B \\
(0, \pm \nu) A & ( \pm \eta, \pm \eta) C .
\end{array}
$$

VII. A formula of degree $\tilde{5}$ for $n=3$ using 13 points:

$$
\begin{array}{lll}
(0,0,0) & A & ( \pm \nu, 0, \pm \xi) B \\
(0, \pm \xi, \pm \nu) B & ( \pm \xi, \pm \nu, 0) B .
\end{array}
$$

VIII. A formula of degree 5 for $n=3$ using 15 points:

$$
\begin{aligned}
& (0,0,0) \quad A \\
& ( \pm \nu, 0,0) B \quad(0,0, \pm \nu) \quad B \\
& (0, \pm \nu, 0) B \quad( \pm \eta, \pm \eta, \pm \eta) C .
\end{aligned}
$$

IX. A formula of degree 5 for $n=3$ using 21 points:

$$
\begin{array}{lll}
(0,0,0) & A & (0, \pm \xi, \pm \nu) B \\
( \pm \eta, \pm \eta, \pm \eta) B & ( \pm \nu, 0, \pm \xi) B \\
& ( \pm \xi, \pm \nu, 0) B .
\end{array}
$$

$\mathrm{X}$. A formula of degree 7 for $n=3$ using 27 points; we use

$$
\begin{array}{ll}
(0,0,0) \quad A & ( \pm \xi, \pm \xi, 0) \quad C^{\gamma} \\
( \pm \nu, 0,0) B & ( \pm \eta, \pm \eta, \pm \eta) D
\end{array}
$$

plus all permutations of the points $( \pm \nu, 0,0)$ and $( \pm \xi, \pm \xi, 0)$.

XI. A formula of degree 7 for $n=3$ using 33 points:

$$
\begin{array}{lll}
(0,0,0) & A & ( \pm \eta, \pm \eta, \pm \eta) C \\
(0, \pm \xi, \pm \nu) B & (0, \pm \lambda, \pm \mu) & C \\
( \pm \nu, 0, \pm \xi) B & ( \pm \mu, 0, \pm \lambda) & C \\
( \pm \xi, \pm \nu, 0) B & ( \pm \lambda, \pm \mu, 0) & C .
\end{array}
$$

All of the above formulas were calculated by solving systems of non-linear equations for the unknown parameters in the manner described in Hammer and Stroud [4].

In the tables $\mathrm{V}$ designates the $n$-dimensional volume or content of the regionthat is the integral of $f=1$ over the region. If $\alpha_{1}, \cdots, \alpha_{n}$ are all non-negative even integers the monomial integrals are

$$
\begin{array}{r}
\int_{-\infty}^{\infty} \cdots \int_{-\infty}^{\infty} \exp \left[-x_{1}{ }^{2}-\cdots-x_{n}{ }^{2}\right] x_{1}^{\alpha_{1}} \cdots x_{n}^{\alpha_{n}} d x_{1} \cdots d x_{n} \\
=\Gamma\left(\frac{\alpha_{1}+1}{2}\right) \Gamma\left(\frac{\alpha_{2}+1}{2}\right) \cdots \Gamma\left(\frac{\alpha_{n}+1}{2}\right) \\
\int_{-\infty}^{\infty} \cdots \int_{-\infty}^{\infty} \exp \left[-\sqrt{{x_{1}{ }^{2}+\cdots+x_{n}{ }^{2}}^{2}} x_{1}^{\alpha_{1}} \cdots x_{n}^{\alpha_{n}} d x_{1} \cdots d x_{n}\right. \\
=2\left(\alpha_{1}+\cdots+\alpha_{n}+n-1\right) ! \frac{\Gamma\left(\frac{\alpha_{1}+1}{2}\right) \cdots \Gamma\left(\frac{\alpha_{n}+1}{2}\right)}{\Gamma\left(\frac{\alpha_{1}+\cdots+\alpha_{n}+n}{2}\right)} .
\end{array}
$$

If one or more of the $\alpha_{i}$ are odd each of these integrals is zero. 
It is interesting to note that the 12 points with coefficient $B$ in formula VII form the vertices of a regular icosahedron and that the 20 points with coefficient $B$ in IX form the vertices of a regular dodecahedron which lie on the surface of the same sphere as the 12 points in VII.

The points in formula VIII with weights $B$ and $C$ all lie on the same sphere as the points in formulas VII and IX. For the integral $U$ an additional solution was found for which the weight of the center point is zero. This then reduces to a 14 point formula. Similarly, the 12 points with coefficient $B$ and the 20 points with coefficient $C$ in XI form the vertices of an icosahedron and its reciprocal dodecahedron-that is the vertices of the dodecahedron lie on rays which pass through the origin and the centroids of the faces of the icosahedron. Formulas of the form VII, IX and XI were given for the sphere by Ditkin [3].

In formula IV for $n=4$ the coefficient $B$ is zero both for $U$ and for $V$. In this case there are 25 points in the formula: the centroid plus 24 points which are the vertices of a regular 4-dimensional polytope which Coxeter designates $\{3,4,3\}$ (Coxeter [2], p. 156).

3. Spherical Product Formulas for $U$ and $V$. Because the region and weight functions in the integrals $U$ and $V$ have the same symmetries as the $n$-dimensional sphere, we can obtain formulas for these integrals by the same spherical product of one-dimensional formulas as Peirce $[10,11]$ used for the circle and 3-sphere and which has been generalized to the $n$-sphere by Hetherington [6].

Before we discuss how this method can be applied to $U$ and $V$, it must be noted that Cartesian product formulas (see, for example, Hammer and Wymore [5]) can also be obtained for $U$. This follows from the fact that when $f\left(x_{1}, \cdots, x_{n}\right)=$ $x_{1}{ }^{\alpha_{1}} \cdots x_{n}{ }^{\alpha_{n}}$ the integral $U$ becomes the product of the $n$ single integrals

$$
\int_{-\infty}^{\infty} e^{-x_{i}{ }^{2}} x_{i}^{\alpha_{i}} d x_{i}, \quad i=1,2, \cdots, n \text {. }
$$

The points in the Cartesian product formulas will be in an $n$-dimensional cubical array whereas in the spherical product formulas the points lie on the surfaces of spheres. Both types of formulas for $U$ will be useful but in a particular case one type will probably be more appropriate than the other.

For an integral in which the region and weight function are both spherically symmetric we can use the transformation between rectangular Cartesian and spherical coordinates

$$
\begin{gathered}
x_{1}=r \cos \theta_{n-1} \cos \theta_{n-2} \cdots \cos \theta_{2} \cos \theta_{1} \\
x_{2}=r \cos \theta_{n-1} \cos \theta_{n-2} \cdots \cos \theta_{2} \sin \theta_{1} \\
x_{3}=r \cos \theta_{n-1} \cos \theta_{n-2} \cdots \sin \theta_{2} \\
\cdots \\
x_{n-1}=r \cos \theta_{n-1} \sin \theta_{n-2} \\
x_{n}=r \sin \theta_{n-1} \\
J=r^{n-1}\left(\cos \theta_{n-1}\right)^{n-2}\left(\cos \theta_{n-2}\right)^{n-3} \cdots\left(\cos \theta_{2}\right)
\end{gathered}
$$


to separate the integral of a monomial $f\left(x_{1}, \cdots, x_{n}\right)=x_{1}{ }^{\alpha_{1}} \cdots x_{n}{ }^{\alpha_{n}}$ into a product of single integrals. For example, for $U$ we obtain:

$$
\begin{array}{rl}
\int e^{-r^{2}} x_{1}^{\alpha_{1}} \cdots x_{n}{ }^{\alpha_{n}} & d x=\int_{-\infty}^{\infty} e^{-r^{2}}|r|^{n-1} r^{\alpha_{1}+\cdots+\alpha_{n}} d r \\
& \times \int_{-\pi / 2}^{\pi / 2}\left(\cos \theta_{1}\right)^{\alpha_{1}}\left(\sin \theta_{1}\right)^{\alpha^{2}} d \theta_{1} \\
& \times \int_{-\pi / 2}^{\pi / 2}\left(\cos \theta_{2}\right)\left(\cos \theta_{2}\right)^{\alpha_{1}+\alpha_{2}}\left(\sin \theta_{2}\right)^{\alpha_{3}} d \theta_{2} \\
\cdots & \times \int_{-\pi / 2}^{\pi / 2}\left(\cos \theta_{n-1}\right)^{n-2}\left(\cos \theta_{n-1}\right)^{\alpha_{1}+\cdots+\alpha_{n-1}}\left(\sin \theta_{n-1}\right)^{\alpha_{n}} d \theta_{n-1} .
\end{array}
$$

We wish to obtain integration formulas which are exact for all polynomials of degree $\leqq k$ (which is the same as requiring it to be exact for all monomials of degree $\leqq k)$. This can be done using quadrature formulas of degree $k$ for each of the single integrals on the right side of (3.2). Formulas obtained in this way we call spherical product formulas.

The formulas which are needed for the single integrals

$$
\int_{-\pi / 2}^{\pi / 2}\left(\cos \theta_{i}\right)^{i-1}\left(\cos \theta_{i}\right)^{\alpha_{1}+\cdots+\alpha_{i}}\left(\sin \theta_{i}\right)^{\alpha_{i+1}} d \theta_{i}, \quad i=1, \cdots, n-1
$$

are independent of the region. These are discussed by Hetherington [6]. His presentation differs slightly from ours in that he would write the first two integrals on the right side of $(3.2)$ as

$$
\int_{0}^{\infty} e^{-r^{2}} r^{\alpha_{1}+\cdots+\alpha_{n}+n-1} d r, \quad \int_{-\pi / 2}^{3 \pi / 2}\left(\cos \theta_{1}\right)^{\alpha_{1}}\left(\sin \theta_{1}\right)^{\alpha_{2}} d \theta_{1}
$$

First we point out his results for the remaining integrals. He shows that the quadrature formulas

$$
\begin{aligned}
\int_{-\pi / 2}^{\pi / 2}\left(\cos \theta_{i}\right)^{i-1}\left(\cos \theta_{i}\right)^{\alpha_{1}+\cdots+\alpha_{i}}\left(\sin \theta_{i}\right)^{\alpha_{i+1}} d \theta_{i} \\
=\sum_{j} B_{i, j}\left(\cos \theta_{i, j}\right)^{\alpha_{1}+\cdots+\alpha_{i}}\left(\sin \theta_{i, j}\right)^{\alpha_{i+1}}, \quad i=2, \cdots, n-1
\end{aligned}
$$

can be taken to coincide with quadrature formulas of the form

$$
\int_{-1}^{1}\left(1-y_{i}^{2}\right)^{(i-2) / 2} y_{i}^{\alpha} d y_{i}=\sum_{j} B_{i, j} y_{i, j}^{\alpha}, \quad i=2, \cdots, n-1
$$

where $y_{i}=\sin \theta_{i}$.

In product formulas we usually want to use one-dimensional formulas of the highest possible accuracy - that is Gaussian type formulas which have the property that an $h$ point formula is exact for polynomials of degree $\leqq 2 h-1$. For $i=2$ the weight function in (3.4) is the identity and the corresponding formula of degree $2 h-1$ is the well-known Gauss-Legendre formula in which the points $y_{2, j}=$ $\sin \theta_{2, j}$ are the $h$ roots of the Legendre polynomial of degree $h$ (Krylov [9] p. 102, or Hildebrand [7] p. 325). For $i=3$ the weight function in (3.4) is $\sqrt{1-y_{3}^{2}}$ and 
the points in the formula of degree $2 h-1$ are the roots of the Chebyshev polynomial of the second kind of degree $h$ (Krylov [9] p. 113). The points and coefficients in this formula are

$$
y_{3, j}=\sin \left(\frac{2 j-h-1}{2 h+2}\right) \pi \quad B_{3, j}=\frac{\pi}{h+1} \sin \frac{j \pi}{h+1} .
$$

In general, the points in the formula (3.4) of the highest degree of accuracy $2 h-1$ are the roots of the Jacobi polynomial $P_{h}{ }^{[(i-2) / 2,(i-2) / 2]}\left(y_{j}\right)$ which is also an ultraspherical or Gegenbauer polynomial.

By the same methods which Hetherington uses to obtain the formulas (3.4) it follows that a quadrature formula for the angular integral

$$
\int_{-\pi / 2}^{\pi / 2}\left(\cos \theta_{1}\right)^{\alpha_{1}}\left(\sin \theta_{1}\right)^{\alpha_{2}} d \theta_{1}=\sum_{j} B_{1, j}\left(\cos \theta_{1, j}\right)^{\alpha_{1}}\left(\sin \theta_{1, j}\right)^{\alpha_{2}}
$$

is given by a formula of the form

$$
\int_{-1}^{1} \frac{1}{\sqrt{1-y_{1}^{2}}} y_{1}^{\alpha} d y_{1}=\sum_{j} B_{1, j} y_{1, j}^{\alpha}
$$

The $h$ points in a formula of this form of the highest degree of accuracy $2 h-1$ are the roots of the Chebyshev polynomial of the first kind of degree $h$; this formula is given explicitly by

$$
y_{1, j}=\sin \theta_{1, j}=\sin \left(\frac{2 j-h-1}{2 h}\right) \pi, \quad B_{1, j}=\frac{\pi}{h}, j=1, \cdots, h
$$

(Krylov [9] p. 111, or Hildebrand [7] p. 331). Hetherington has tabulated the points and coefficients in (3.4) for $i=2(1) 7, h=1(1) 8$.

To construct spherical product formulas for $U$ and $V$ there remains only to have formulas of the forms

$$
\begin{aligned}
& \int_{-\infty}^{\infty}|r|^{n-1} e^{-r^{2}} r^{\alpha} d r=\sum_{j} B_{j} r_{j}^{\alpha} \\
& \int_{-\infty}^{\infty}|r|^{n-1} e^{-|r|} r^{\alpha} d r=\sum_{j} B_{j} r_{j}^{\alpha} .
\end{aligned}
$$

In the following section we discuss formulas of these types and tabulate some of these formulas of the highest degree of accuracy $2 h-1$. The spherical product formulas of degree $2 h-1$ which are derived by combining one of these $h$ point formulas with $h$ point formulas (3.6) and (3.4), for $i=2, \cdots, n-1$, will contain $h^{n}$ points for $h$ even and $h^{n}-h^{n-1}+1$ points for $h$ odd. For even $h$ the points lie on the surfaces of $h / 2$ distinct spheres with $2 h^{n-1}$ points on each sphere; for $h$ odd there are $2 h^{n-1}$ points on the surfaces of $(h-1) / 2$ spheres, and in addition, the center of the sphere is also a point in the formula. In Section 5 we give examples which illustrate the construction and use of spherical product formulas.

4. The Quadrature Formulas (3.8) and (3.9). From the theory of orthogonal polynomials it is known that the points in formulas (3.8) and (3.9) are the roots of polynomials $M_{h}{ }^{(n)}(r)$ and $N_{h}{ }^{(n)}(r)$ of degree $h$ which are orthogonal to all polynomials of degree $<h$ on $(-\infty, \infty)$ with respect to $|r|^{n-1} e^{-r^{2}}$ and $|r|^{n-1} e^{-|r|}$ 
respectively. The polynomials $M_{h}^{(n)}$ and $N_{h}{ }^{(n)}$ are related to polynomials $L_{m}{ }^{(\alpha)}$ and $K_{m}{ }^{(\alpha)}$ which are orthogonal on $[0, \infty)$. It is these latter polynomials which we will investigate.

The relationships which we desire between the orthogonal polynomials on $(-\infty, \infty)$ and those on $[0, \infty)$ are special cases of a more general theorem. Let $P_{h}{ }^{(\alpha, \beta)}(r)$ be the polynomial which satisfies the orthogonality conditions

$$
\int_{-\infty}^{\infty}|r|^{2 \alpha+1} e^{-r \beta} P_{h}^{(\alpha, \beta)}(r) r^{i} d r=0, \quad i=0,1, \cdots, h-1
$$

and let $Q_{m}{ }^{(\alpha, \beta)}(x)$ satisfy the orthogonality conditions

$$
\int_{0}^{\infty} x^{\alpha} e^{-\sqrt{x^{\beta}}} Q_{m}{ }^{(\alpha, \beta)}(x) x^{i} d x=0, \quad i=0,1, \cdots, m-1
$$

where we assume $\alpha \geqq 0, \beta>0$.

Theorem. The $P_{h}{ }^{(\alpha, \beta)}$ and $Q_{m}{ }^{(\alpha, \beta)}$ are related in the following way:

$$
\begin{aligned}
& P_{2 m}^{(\alpha, \beta)}(r)=Q_{m}{ }^{(\alpha, \beta)}\left(r^{2}\right) \\
& P_{2 m+1}^{(\alpha, \beta)}(r)=r Q_{m}^{(\alpha+1, \beta)}\left(r^{2}\right) .
\end{aligned}
$$

Proof. Let us first prove (4.3). We must show that

$$
\int_{-\infty}^{\infty}|r|^{2 \alpha+1} e^{-r \beta} Q_{m}{ }^{(\alpha, \beta)}\left(r^{2}\right) r^{i} d r=0, \quad i=0,1, \cdots, 2 m-1 .
$$

If $i$ is an odd integer then the integrand is odd and thus (4.5) is identically satisfied. If $i=2 j$, using the transformation $r^{2}=x$, we obtain

$$
\begin{aligned}
& \int_{-\infty}^{\infty}|r|^{2 \alpha+1} e^{-r \beta} Q_{m}{ }^{(\alpha, \beta)}\left(r^{2}\right) r^{2 j} d r=\int_{0}^{\infty} x^{\alpha} e^{-\sqrt{x^{\beta}}} Q_{m}{ }^{(\alpha, \beta)}(x) x^{j} d x=0, \\
& j=0,1, \cdots, m-1
\end{aligned}
$$

by $(4.2)$.

To prove (4.4) we must show that

$$
\int_{-\infty}^{\infty}|r|^{2 \alpha+1} e^{-r \beta} r Q_{m}{ }^{(\alpha+1, \beta)}\left(r^{2}\right) r^{i} d r=0, \quad i=0,1, \cdots, 2 m .
$$

In this case (4.6) is identically satisfied if $i$ is an even integer. For $i=2 j+1$

$$
\begin{aligned}
\int_{-\infty}^{\infty}|r|^{2 \alpha+1} e^{-r \beta} r Q_{m}{ }^{(\alpha+1, \beta)} & \left(r^{2}\right) r^{2 j+1} d r \\
& =\int_{0}^{\infty} x^{\alpha+1} e^{-\sqrt{r^{\beta}}} Q_{m}{ }^{(\alpha+1, \beta)}(x) x^{j} d x=0, \quad j=0,1, \cdots, m-1
\end{aligned}
$$

by (4.2). This completes the proof.

Thus for $\beta=2$ the $Q_{m}{ }^{(\alpha, 2)}(x)$ coincide with the Laguerre polynomials $L_{m}{ }^{(\alpha)}(x)$ (Szegö [15], p. 102):

$$
\begin{aligned}
M_{2 m}^{(n)}(r) & =L_{m}{ }^{[(n-2) / 2]}\left(r^{2}\right) \\
M_{2 m+1}^{(n)}(r) & =r L_{m}{ }^{(n / 2)}\left(r^{2}\right) .
\end{aligned}
$$


For $\beta=1$ we change notation and write $K_{m}{ }^{(\alpha)}(x)={Q_{m}}^{(\alpha, 1)}(x)$. Then

$$
\begin{aligned}
N_{2 m}^{(n)}(r) & =K_{m}{ }^{[(n-2) / 2]}\left(r^{2}\right) \\
N_{2 m+1}^{(n)}(r) & =r K_{m}{ }^{(n / 2)}\left(r^{2}\right) .
\end{aligned}
$$

The $K_{m}{ }^{(\alpha)}(x)$ have not been investigated previously. In Table 3 we tabulate these polynomials for $\alpha=0\left(\frac{1}{2}\right) 2, \quad m=1(1) 10$.

From this theorem it is easy to see that there is a relationship between the quadrature formulas

$$
\int_{-\infty}^{\infty}|r|^{n-1} e^{-r \beta} f(r) d r \approx \sum_{k=1}^{h} B_{k}^{(n)} f\left(r_{k}^{(n)}\right)
$$

and the formulas

$$
\int_{0}^{\infty} x^{\alpha} e^{-\sqrt{x^{\beta}}} f(x) d x \approx \sum_{k=1}^{m} A_{k}^{(\alpha)} f\left(x_{k}^{(\alpha)}\right)
$$

(We assume that these formulas are exact for polynomials of degrees $\leqq 2 h-1$ and $\leqq 2 m-1$ respectively.) The $x_{k}{ }^{(\alpha)}(k=1, \cdots, m)$ are the zeros of $Q_{m}{ }^{(\alpha, \beta)}(x)$. To simplify the discussion we relabel the points and coefficients in (4.7) for $h=2 m$ as

$$
\begin{aligned}
& r_{-m}^{(2 m)}, \cdots, r_{-1}^{(2 m)}, r_{1}{ }^{(2 m)}, \cdots, r_{m}{ }^{(2 m)} \\
& B_{-m}^{(2 m)}, \cdots, B_{-1}^{(2 m)}, B_{1}{ }^{(2 m)}, \cdots, B_{m}{ }^{(2 m)}
\end{aligned}
$$

and for $h=2 m+1$ as

$$
\begin{aligned}
& r_{-m}^{(2 m+1)}, \cdots, r_{-1}^{(2 m+1)}, r_{0}{ }^{(2 m+1)}, r_{1}{ }^{(2 m+1)}, \cdots, r_{m}{ }^{(2 m+1)} \\
& B_{-m}^{(2 m+1)}, \cdots, B_{-1}^{(2 m+1)}, B_{0}{ }^{(2 m+1)}, B_{1}{ }^{(2 m+1)}, \cdots, B_{m}{ }^{(2 m+1)} .
\end{aligned}
$$

Then for even $h$ we have the relationship

$$
\begin{aligned}
-r_{-j}^{(2 m)} & =r_{j}{ }^{(2 m)}=\sqrt{x_{j}^{[(n-2) / 2]}} \\
B_{-j}^{(2 m)} & =B_{j}{ }^{(2 m)}=\left(\frac{1}{2}\right) A_{j}{ }^{[(n-2) / 2]} \quad j=1,2, \cdots, m .
\end{aligned}
$$

For odd $h$ we have

$$
\begin{aligned}
-r_{-j}^{(2 m+1)}= & r_{j}{ }^{(2 m+1)}=\sqrt{x_{j}^{(n / 2)}} \\
B_{-j}^{(2 m+1)}=B_{j}{ }^{(2 m+1)}= & A_{j}{ }^{(n / 2)} / 2 x_{j}{ }^{(n / 2)} j=1,2, \cdots, m \\
& r_{0}{ }^{(2 m+1)}=0 \\
B_{0}{ }^{(2 m+1)}= & \int_{-\infty}^{\infty}|r|^{n-1} e^{-r \beta} d r-2\left(B_{1}{ }^{(2 m+1)}+\cdots+B_{m}{ }^{(2 m+1)}\right) \\
= & \frac{2}{\beta} \Gamma\left(\frac{n}{\beta}\right)-2\left(B_{1}{ }^{(2 m+1)}+\cdots+B_{m}{ }^{(2 m+1)}\right) .
\end{aligned}
$$

Relationships (4.9) and (4.10) are readily verified by comparing the systems of equations which must be satisfied from the requirement that formulas (4.7) be exact for the monomials $f(r)=1, r, r^{2}, \cdots, r^{2 h-1}$ and that formula (4.8) be exact for the monomials $f(x)=1, x, x^{2}, \cdots, x^{2 m-1}$. Since the points and coefficients 
TABle 3

The Polynomials* $K_{m}^{(\alpha)}(x)$

\begin{tabular}{|c|c|c|c|}
\hline \multicolumn{4}{|c|}{$\alpha=0$} \\
\hline$x$ & 6.000000000 & & \\
\hline$x^{2}$ & $51.42857143 x+$ & 188.5714286 & \\
\hline$x^{3}$ & $175.7980456 x^{2}+$ & $5081.042345 x$ & 14430.48860 \\
\hline$x^{4}$ & $\begin{array}{l}418.5938754 x^{3}+ \\
2039377.990\end{array}$ & $39998.92982 x^{2}$ & $848736.0728 x$ \\
\hline$x^{5}$ & $\begin{array}{l}819.2973711 x^{4}+ \\
216564550.9 x-\end{array}$ & $\begin{array}{l}183018.2465 x^{3}- \\
460569374.5\end{array}$ & $12532000.53 x^{2}$ \\
\hline$x^{6}$ & $\begin{array}{l}1417.388499 x^{5}+ \\
5290764177 \cdot x^{2}-\end{array}$ & $\begin{array}{rr}612504.1485 x^{4} \\
-\quad 7861311062(1) x\end{array}$ & $\begin{array}{r}96656694.23 x^{3} \\
+\quad 1520217885(2)\end{array}$ \\
\hline$x^{7}$ & $\begin{array}{l}-\quad 2252.346696 x^{6}+ \\
+\quad 6298366037(1) x^{3}- \\
-\quad 6902314537(4)\end{array}$ & $\begin{array}{r}1670231.460 x^{5} \\
+\quad 2920496077(3) x^{2}\end{array}$ & $\begin{array}{rr}505059472.7 x^{4} \\
+\quad 3856140801(4) x\end{array}$ \\
\hline$x^{8}$ & $\begin{array}{rr} & 3363.651265 x^{7}+ \\
+ & 4814328148(2) x^{4}- \\
- & 2459122665(7) x+\end{array}$ & $\begin{array}{l}+\quad 3940091.323 x^{6}- \\
+\quad 5037579765(4) x^{3}- \\
+\quad 4125679289(7)\end{array}$ & $\begin{array}{rr}-\quad 2030683982 & x^{5} \\
+\quad 2050404135(6) x^{2}\end{array}$ \\
\hline$x^{9}$ & $\begin{array}{rr}-\quad 4790.780103 x^{8}+ \\
+\quad 2714601855(3) x^{5}- \\
-\quad 1788203448(9) x^{2}+\end{array}$ & $\begin{array}{r}8342379.300 x^{7} \\
+\quad 5363905256(5) x^{4} \\
+\quad 1978574432(10) x\end{array}$ & $\begin{array}{lr} & 6757097345 \cdot x^{6} \\
+ & 4887089022(7) x^{3} \\
- & 3140231892(10)\end{array}$ \\
\hline$x^{10}$ & $\begin{array}{l}-\quad 6573.192474 x^{9}+ \\
+\quad 1224496326(4) x^{6}- \\
-5675915473(10) x^{3}+ \\
+2965220249(13)\end{array}$ & 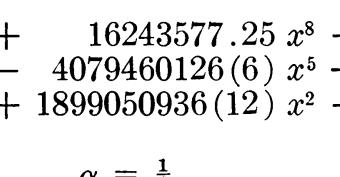 & $\begin{array}{r}-\quad 1948082357(1) x^{7} \\
+\quad 6978110058(8) x^{4} \\
-1960983943(13) x\end{array}$ \\
\hline$x$ & 12.00000000 & $\alpha-2$ & \\
\hline$x^{2}$ & $73.33333333 x+$ & 520.0000000 & \\
\hline$x^{3}$ & $223.4862385 x^{2}+$ & $9108.990826 x$ & 49012.84404 \\
\hline$x^{4}$ & $\begin{array}{l}501.9397772 x^{3}+ \\
8078803.906\end{array}$ & $60629.91718 x^{2}$ & $1800072.349 x$ \\
\hline$x^{5}$ & $\begin{array}{l}948.1736367 x^{4}+ \\
524334762.9 x\end{array}$ & $\begin{array}{l}253105.4084 x^{3} \\
2060786564 .\end{array}$ & $21813806.05 x^{2}$ \\
\hline$x^{6}$ & $\begin{array}{lr}- & 1601.666964 x^{5}+ \\
+ & 1031828719(1) x^{2}-\end{array}$ & $\begin{array}{rr}799149.3167 x^{4} \\
-\quad 2122585065(2) x\end{array}$ & $\begin{array}{l}-\quad 150283152.2 x^{3} \\
+\quad 7522618887(2)\end{array}$ \\
\hline$x^{7}$ & $\begin{array}{lr}- & 2501.898648 x^{6}+ \\
+ & 1081390288(2) x^{3}- \\
- & 3721377483(5)\end{array}$ & $\begin{array}{r}2092983.870 x^{5} \\
+\quad 6271861623(3) x^{2}\end{array}$ & $\begin{array}{rr}729338100.8 x^{4} \\
+\quad 1142132567(5) x\end{array}$ \\
\hline$x^{8}$ & $\begin{array}{rr}- & 3688.347161 x^{7}+ \\
+ & 7590364740(2) x^{4}- \\
- & 7892790318(7) x+\end{array}$ & $\begin{array}{rr}+ & 4793041.659 x^{6}- \\
+ & 9424985629(4) x^{3} \\
+ & 2396782720(8)\end{array}$ & $\begin{array}{rr}2782802603 . x^{5} \\
+\quad 4786891163(6) x^{2}\end{array}$ \\
\hline$x^{9}$ & $\begin{array}{rr}- & 5200.488021 x^{8}+ \\
+ & 4024764407(3) x^{5}- \\
- & 4493788769(9) x^{2}+\end{array}$ & 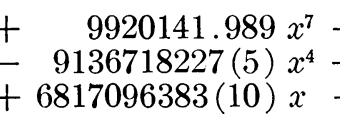 & $\begin{array}{lr} & 8904201671 \cdot x^{6} \\
+ & 9862174618(7) x^{3} \\
- & 1948908494(11)\end{array}$ \\
\hline$x^{10}$ & $\begin{array}{l}-\quad 7077.780814 x^{9}+ \\
+\quad 1732881036(4) x^{6}- \\
-1225474423(11) x^{3}+ \\
+1952611616(14)\end{array}$ & $\begin{array}{r}18971168.02 x^{8} \\
+\quad \begin{array}{r}648498949(6) \\
x^{5}\end{array}-5096958559(12) x^{2}\end{array}$ & $\begin{array}{rr}- & 2490180057(1) x^{7} \\
+\quad 1273559512(9) & x^{4} \\
- & 7198987016(13) x\end{array}$ \\
\hline
\end{tabular}

* The coefficients are exact to 10 significant figures. The powers of 10 by which some of the coefficients must be multiplied are written in parentheses : $1520217885(2)=1520217885 . \times 10^{2}$. 
TABle 3 (Continued)

\begin{tabular}{|c|c|c|c|}
\hline \multicolumn{4}{|c|}{$\alpha=1$} \\
\hline$x$ & 20.00000000 & & \\
\hline$x^{2}$ & $99.27272727 x$ & 1145.454545 & \\
\hline$x^{3}$ & $277.2696246 x^{2}$ & $15154.40273 x$ & 130661.5700 \\
\hline$x^{4}$ & $\begin{array}{l}593.4592101 x^{3}+ \\
24887557.15\end{array}$ & $88483.77552 x^{2}-$ & $3498715.778 x$ \\
\hline$x^{5}$ & $\begin{array}{l}1087.314904 x^{4} \dashv \\
1154281719 . x\end{array}$ & $\begin{array}{l}342075.7196 x^{3}- \\
7130871015 .\end{array}$ & $36247203.68 x^{2}$ \\
\hline$x^{6}$ & $\begin{array}{rr} & 1798.312133 x^{5}+ \\
+ & 1909818061(1) x^{2}\end{array}$ & $\begin{array}{r}1026688.091 x^{4}- \\
5183062928(2) x+\end{array}$ & $\begin{array}{lc} & 226961419.7 x^{3} \\
+ & 2868339052(3)\end{array}$ \\
\hline$x^{7}$ & $\begin{array}{lr}- & 2765.927266 x^{6}+ \\
+ & 1795466367(2) x^{3} \\
- & 1542146359(6)\end{array}$ & $\begin{array}{r}2593809.633 x^{5}- \\
1272895065(4) x^{2}+\end{array}$ & $\begin{array}{rr}- & 1032346531 \\
+ & 3047534514(5)\end{array} x^{4}$ \\
\hline$x^{8}$ & $\begin{array}{lr}- & 4029.637402 x^{7} \\
+ & 1168961349(3) x^{4} \\
- & 2275528327(8) x\end{array}$ & $\begin{array}{r}5782160.585 x^{6} \\
-\quad 1699513817(5) x^{3}+ \\
-\quad 1068305070(9)\end{array}$ & $\begin{array}{rr}- & 3758125331 . x^{5} \\
+ & 1052770996(7) x^{2}\end{array}$ \\
\hline$x^{9}$ & $\begin{array}{l}-\quad 5628.925604 x^{8} \\
+\quad 5864458401(3) x^{5} \\
-1061196079(10) x^{2}\end{array}$ & $\begin{array}{r}11719801.98 x^{7} \\
-21516150533(6) x^{4}+\end{array}$ & $\begin{array}{l}-1160338991(1) x^{6} \\
+\quad 1913149358(8) x^{3} \\
-9267980415(11)\end{array}$ \\
\hline$x^{10}$ & $\begin{array}{l}-\quad 7603.325254 x^{9}+ \\
+\quad 2419799785(4) x^{6} \\
-2538390644(11) x^{3} \\
+9843784288(14)\end{array}$ & 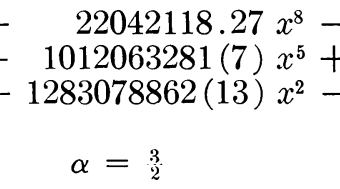 & $\begin{array}{r}-\quad 3155377703(1) x^{7} \\
+\quad 2259648072(9) x^{4} \\
-2364615087(14) x\end{array}$ \\
\hline$x$ & 30.00000000 & & \\
\hline$x^{2}$ & $129.2307692 x$ & 2196.923077 & \\
\hline$x^{3}$ & $337.1094339 x^{2} \dashv$ & $23792.60377 x-$ & 298634.2640 \\
\hline$x^{4}$ & $\begin{array}{l}693.0880340 x^{3} \\
65162222.89\end{array}$ & $125067.7978 x^{2}-$ & $6347987.083 x$ \\
\hline$x^{5}$ & $\begin{array}{l}1236.630670 x^{4} t \\
2355456285 . x\end{array}$ & $\begin{array}{c}453031.5265 x^{3}- \\
-\quad 2085728806(1)\end{array}$ & $57890491.98 x^{2}$ \\
\hline$x^{6}$ & $\begin{array}{rr} & 2007.206891 x^{5}+ \\
+ & 3379519298(1) x^{2}\end{array}$ & $\begin{array}{r}1300652.427 x^{4}- \\
1167490071(3) x+\end{array}$ & $\begin{array}{l}-\quad 334005523.8 x^{3} \\
+\quad 9210724912(3)\end{array}$ \\
\hline$x^{7}$ & $\begin{array}{lr}- & 3044.289152 x^{6}+ \\
+ & 2893004487(2) x^{3} \\
- & 5367947224(6)\end{array}$ & $\begin{array}{r}3181673.818 x^{5} \\
2460205275(4) x^{2}+\end{array}$ & $\begin{array}{rr}- & 1434799615 \\
+ & 7474487576(5) \\
x^{4}\end{array}$ \\
\hline$x^{8}$ & $\begin{array}{rr}- & 4387.352674 x^{7} \\
+ & 1761969265(3) x^{4} \\
- & 6013986384(8) x\end{array}$ & $\begin{array}{rr}6921016.533 x^{6} \\
-\quad 2964724499(5) x^{3} \\
+\quad 3991902394(9)\end{array}$ & $\begin{array}{rr}5006810077 & x^{5} \\
+ & 2198385994(7) x^{2}\end{array}$ \\
\hline$x^{9}$ & $\begin{array}{l}-\quad 6075.882052 x^{8} \\
+\quad 8407696631(3) x^{5} \\
-2373833208(10) x^{2}\end{array}$ & $\begin{array}{r}13760804.51 x^{7} \\
-\quad 2456089445(6) x^{4} \\
-5946318360(11) x\end{array}$ & $\begin{array}{l}-\quad 1496281879(1) x^{6} \\
+\quad 3581520053(8) x^{3} \\
-3689378949(12)\end{array}$ \\
\hline$x^{10}$ & $\begin{array}{l}-\quad 8149.406551 x^{9}+ \\
+\quad 3336338166(4) x^{6} \\
-5063537526(11) x^{3} \\
+4148914598(15)\end{array}$ & $\begin{array}{r}25482243.54 x^{8} \\
1549842529(7) x^{5} \\
-3053567283(13) x^{2}\end{array}$ & $\begin{array}{r}-\quad 3964825086(1) x^{7} \\
+\quad 3905576272(9) x^{4} \\
-7092156231(14) x\end{array}$ \\
\hline
\end{tabular}


TABLE 3 (Continued)

$\alpha=2$

$$
\begin{aligned}
& x \quad-42.00000000 \\
& x^{2}-163.20000000 x+3830.400000 \\
& x^{3}-402.9857820 x^{2}+35671.27962 x-612204.7393 \\
& x^{4}-800.7913617 x^{3}+172035.6848 x^{2}-10894518.93 x \\
& +\quad 151817282.9 \\
& x^{5}-1396.069675 x^{4}+589323.8733 x^{3}-89364042.12 x^{2} \\
& +\quad 4518016412 . x-5400518134(1) \\
& x^{6}-2228.282870 x^{5}+1626957.197 x^{4}-480353438.3 x^{3} \\
& +5752210325(1) x^{2}-2460921109(3) x+2608898621(4) \\
& x^{7}-3336.898278 x^{6}+3866094.552 x^{5}-1961291918 . x^{4} \\
& +4538145202(2) x^{3}-4557240322(4) x^{2}+1709868012(6) x \\
& \text { - } 1643820045(7) \\
& x^{8}-4761.385316 x^{7}+8223928.986 x^{6}-6587132059 . x^{5} \\
& +2604132694(3) x^{4}-5020292312(5) x^{3}+4387522327(7) x^{2} \\
& \text { - 1478745271(9) } x+1309692475(10) \\
& x^{9}-6541.198703 x^{8}+16063486.19 x^{7}-1910586079(1) x^{6} \\
& +1187372390(4) x^{5}-3891831885(6) x^{4}+6493029802(8) x^{3} \\
& -5063817639(10) x^{2}+1559528933(12) x-1287478156(13) \\
& x^{10}-8715.671825 x^{9}+\quad 29318198.51 x^{8}-4942194283(1) x^{7} \\
& +4545034140(4) x^{6}-2332833395(7) x^{5}+6588716467(9) x^{4} \\
& -9760896083(11) x^{3}+6915805277(13) x^{2}-1971200587(15) x \\
& +1530562149(16)
\end{aligned}
$$

in (4.7) are symmetric with respect to $r=0$ we must remember that (4.7) will be exact identically for $f(r)=r, r^{3}, \cdots, r^{2 h-1}$.

In Table 4 we tabulate the $r_{k}$ and $B_{k}$ in formula (3.8) for $n=2(1) 4$. In Table 5 we give the formulas (3.9) for $n=2(1) 4$. The $r_{k}$ and $B_{k}$ are given to $10 \mathrm{~S}$ and since they are symmetric we tabulate only the values for $0 \leqq r_{k}$. These formulas were calculated from the corresponding formulas for the interval $[0, \infty)$ using (4.9) and (4.10).

The polynomials $L_{m}{ }^{(\alpha)}(x)$ and $K_{m}{ }^{(\alpha)}(x)$ were calculated sequentially using the recurrence relation

$$
\begin{gathered}
Q_{0}(x)=1, \quad Q_{1}(x)=x-a \\
Q_{n+1}(x)=\left(x+b_{n}\right) Q_{n}(x)+c_{n} Q_{n-1}(x), \quad n=1,2, \cdots
\end{gathered}
$$

(where $a=\alpha+1$ for $L_{m}{ }^{(\alpha)}$ and $a=(2 \alpha+3)(2 \alpha+2)$ for $K_{m}{ }^{(\alpha)}$ ) and calculating the $b_{n}$ and $c_{n}$ from the monomial integrals. The roots of these polynomials were found by Newton-Raphson iteration. The $A_{k}{ }^{(\alpha)}(k=1, \cdots, m)$ in (4.8) were determined as the solution of a linear system of equations. Each of the resulting formulas (4.8) were checked by computing all of the monomial integrals for which the formula should be exact. The largest discrepancy in any of these integrals was 1 in the 10-th significant figure. In a few cases it was possible to check the formulas corresponding to the Laguerre polynomials with the formulas given by Rabino- 
TABLE 4.

Quadrature Formulas for the Integral $\int_{-\infty}^{\infty}|r|^{n-1} e^{-r^{2}} f(r) d r$.

\begin{tabular}{|c|c|c|c|}
\hline & & $n=2$ & \\
\hline$r_{i}$ & $h=2$ & $r_{i}$ & $h=12$ \\
\hline $1.000000000(0)$ & $5.000000000(-1)$ & $4.720663133(-1)$ & $2.294823369(-1)$ \\
\hline & $h=3$ & $1.090381631(0)$ & $2.085004154(-1)$ \\
\hline $0.000000000(0)$ & $5.000000000(-1)$ & $\begin{array}{l}1.729952695(0) \\
2.403152839(0)\end{array}$ & $\begin{array}{l}5.6686699103(-2) \\
5.199598727(-3)\end{array}$ \\
\hline $1.414213562(0)$ & $2.500000000(-1)$ & $3.136473724(0)$ & $1.305086014(-4)$ \\
\hline & $h=4$ & $3.997858674(0)$ & $4.492739532(-7)$ \\
\hline $7.653668647(-1)$ & $4.267766953(-1)$ & & $h=13$ \\
\hline $1.847759065(0)$ & $7.322330470(-2)$ & $0.000000000(0)$ & $1.428571428(-1)$ \\
\hline & $h=5$ & $\begin{array}{l}7.264076828(-1) \\
1.340261098(0)\end{array}$ & $\begin{array}{l}2.630916391(-1) \\
1.374799965(-1)\end{array}$ \\
\hline $0.000000000(0)$ & $3.333333333(-1)$ & $1.968918871(0)$ & $2.618301121(-2)$ \\
\hline $1.126032501(0)$ & $3.110042340(-1)$ & $2.630364341(0)$ & $1.782734094(-3)$ \\
\hline $2.175327747(0)$ & $2.232909937(-2)$ & $3.351807039(0)$ & $3.395946714(-5)$ \\
\hline & $h=6$ & $4.200709887(0)$ & $\begin{array}{r}8.826492752(-8) \\
-6\end{array}$ \\
\hline $6.448058287(-1)$ & 3.555465050 (-1) & $4.393673595(-1)$ & $\begin{aligned} h= & 14 \\
& 2.046594758(-1)\end{aligned}$ \\
\hline $1.514688206(0)$ & $1.392588668(-1)$ & $1.013244736(0)$ & $2.109156390(-1)$ \\
\hline $2.507976292(0)$ & $5.194628251(-3)$ & $1.602459592(0)$ & $7.356317431(-2)$ \\
\hline & $h=7$ & $2.213674114(0)$ & $1.031675724(-2)$ \\
\hline $0.000000000(0)$ & $2.500000000(-1)$ & $2.860446372(0)$ & 5.370050716 (-4) \\
\hline $9.673790506(-1)$ & $3.145263404(-1)$ & $3.568498324(0)$ & $\begin{array}{l}7.932732175(-6) \\
158515739(-8)\end{array}$ \\
\hline $1.818077911(0)$ & $5.917819272(-2)$ & $4.404058113(0)$ & $h=1.585157739(-8)$ \\
\hline $2.785456961(0)$ & $1.295466838(-3)$ & $0.000000000(0)$ & $n=\frac{10}{1.250000000(-1)}$ \\
\hline & $h=8$ & $6.789876433(-1)$ & $2.453348293(-1)$ \\
\hline $5.679328214(-1)$ & $3.015770522(-1)$ & $1.250434400(0)$ & $1.502023715(-1)$ \\
\hline & $1.787093462(-1)$ & $1.830860591(0)$ & $3.775636974(-2)$ \\
\hline $41(0)$ & $1.944395426(-2)$ & $2.432343982(0)$ & $4.039951583(-3)$ \\
\hline $3.065137992(0)$ & $2.696473528(-4)$ & $3.069315784(0)$ & $1.645557103(-4)$ \\
\hline & $h=9$ & $3.767514505(0)$ & 1. $919262837(-6)$ \\
\hline $0.000000000(0)$ & $2.000000000(-1)$ & $4.5926220008(0)$ & $\begin{aligned} h= & 2.994806763(-9) \\
h= & 16\end{aligned}$ \\
\hline $8.621437977(-1)$ & $3.006023451(-1)$ & $4.126495272(-1)$ & $1.845942946(-1)$ \\
\hline $1.603631818(0)$ & $9.286616703(-2)$ & $9.506323036(-1)$ & $2.093933904(-1)$ \\
\hline $\begin{array}{l}2.393988043(0) \\
3.309666798(0)\end{array}$ & $\begin{array}{l}6.471424810(-3) \\
6.006309941(-5)\end{array}$ & $1.500362166(0)^{-1}$ & $8.789749333(-2)$ \\
\hline & $h=10$ & $\begin{array}{l}2.065599228(0) \\
2.654412440(0)\end{array}$ & $\begin{array}{l}1.667174613(-2) \\
1.397268118(-3)\end{array}$ \\
\hline $5.133812616(-1)$ & $2.608778053(-1)$ & $3.280017684(0)$ & $4.538254387(-5)$ \\
\hline $1.188866291(0)$ & $1.993334055(-1)$ & $3.967452412(0)$ & $4.242873358(-7)$ \\
\hline $1.896424470(0)$ & $3.797122484(-2)$ & $4.781540728(0)$ & $5.240005876(-10)$ \\
\hline $2.661918482(0)$ & $1.805879340(-3)$ & & $h=17$ \\
\hline $3.555390393(0)$ & $1.168498619(-5)$ & $0.000000000(0)$ & $1.111111122(-1)$ \\
\hline & $h=11$ & $6.398308942(-1)$ & $2.291647162(-1)$ \\
\hline $0.000000000(0)$ & $1.666666667(-1)$ & $\begin{array}{l}1.180844588(0) \\
1.719376211(0)\end{array}$ & $\begin{array}{l}1.584826800(-1) \\
4.904785558(-2)\end{array}$ \\
\hline $7.855131146(-1)$ & $2.820074054(-1)$ & $2.276388170(0)$ & $7.250309699(-3)$ \\
\hline $1.453604471(0)$ & $1.188567833(-1)$ & $2.856870611(0)$ & $4.859672146(-4)$ \\
\hline $2.147285065(0)$ & 1. $528096061(-2)$ & $3.474198487(0)$ & $1.278544852(-5)$ \\
\hline $2.898114382(0)$ & $5.190989104(-4)$ & $4.153280092(0)$ & $9.707274072(-8)$ \\
\hline $3.776255164(0)$ & $2.418402001(-6)$ & $4.958422657(0)$ & $9.601808801(-11)$ \\
\hline & & $n=3$ & \\
\hline$r_{i}$ & $B_{i}$ & $r_{i}$ & $B_{i}$ \\
\hline & $h=2$ & & $h=4$ \\
\hline $1.224744871(0)$ & $4.431134628(-1)$ & $9.585724646(-1)$ & $3.616815118(-1)$ \\
\hline & $h=3$ & & $h=5$ \\
\hline & 3.544907702 & $0.000000000(0)$ & $2.025661544(-1)$ \\
\hline 1.58 & $2.658680777(-1)$ & $1.276389951(0)$ & $3.130276580(-1)$ \\
\hline & & $2.317504842(0)$ & $2.880272754(-2)$ \\
\hline
\end{tabular}


TABLE 4. (Continued)

\begin{tabular}{|c|c|c|c|}
\hline \multicolumn{4}{|c|}{$n=3$} \\
\hline$r_{i}$ & $B_{i}$ & $r_{i}$ & $B_{i}$ \\
\hline \multicolumn{2}{|c|}{$h=6$} & \multicolumn{2}{|c|}{$h=15$} \\
\hline $8.162878829(-1)$ & $2.835931389(-1)$ & \multirow{8}{*}{$\begin{array}{l}0.000000000(0) \\
7.849498020(-1) \\
1.358059318(0) \\
1.936174209(0) \\
2.534259912(0) \\
3.167438808(0) \\
3.861603548(0) \\
4.682268068(0)\end{array}$} & \multirow{8}{*}{$\begin{array}{l}4.977355659(-2) \\
1.928583232(-1) \\
1.669603915(-1) \\
5.172219818(-2) \\
6.392121794(-3) \\
2.899586660(-4) \\
3.684908302(-6) \\
6.192708444(-9)\end{array}$} \\
\hline $1.673551629(0)$ & $1.526858844(-1)$ & & \\
\hline $2.651961357(0)$ & $6.834439385(-3)$ & & \\
\hline & & & \\
\hline & $=7$ & & \\
\hline $0.000000000(0)$ & $1.350441030(-1)$ & & \\
\hline $1.104718207(0)$ & $2.993430461(-1)$ & & \\
\hline $1.951635397(0)$ & $7.433274262(-2)$ & & \\
\hline $2.910449615(0)$ & $1.915622567(-3)$ & \multicolumn{2}{|c|}{$h=16$} \\
\hline \multicolumn{2}{|c|}{$h=8$} & \multirow{7}{*}{$\begin{array}{l}5.316330013(-1) \\
1.067648726(0) \\
1.612924314(0) \\
2.173502827(0) \\
2.757762916(0) \\
3.378932091(0) \\
4.061946676(0) \\
4.871345194(0)\end{array}$} & \multirow{7}{*}{$\begin{array}{l}1.135696806(-1) \\
1.967972719(-1) \\
1.064544853(-1) \\
2.393874162(-2) \\
2.271258737(-3) \\
8.120230012(-5) \\
8.211887067(-7) \\
1.086971564(-9)\end{array}$} \\
\hline $7.235510187(-1)$ & $2.265043733(-1)$ & & \\
\hline $1.468553289(0)$ & $1.908084801(-1)$ & & \\
\hline $2.266580585(0)$ & $2.539731379(-2)$ & & \\
\hline $3.190993202(0)$ & $4.032955751(-4)$ & & \\
\hline & $=9$ & & \\
\hline $0.000000000(0)$ & $9.821389311(-2)$ & & \\
\hline $9.891952629(-1)$ & $2.712651779(-1)$ & \multicolumn{2}{|c|}{$h=17$} \\
\hline $1.729270754(0)$ & $1.132309532(-1)$ & $0.000000000(0)$ & $4.191457215(-2)$ \\
\hline $2.513821891(0)$ & $9.411546469(-3)$ & $7.407712334(-1)$ & $1.731693172(-1)$ \\
\hline $3.422252897(0)$ & $9.883855140(-5)$ & $1.279914488(0)$ & $1.710549742(-1)$ \\
\hline \multicolumn{2}{|c|}{$h=10$} & $1.820724044(0)$ & $6.576202749(-2)$ \\
\hline $6.568095669(-1)$ & $1.852252850(-1)$ & $2.374934336(0)$ & $1.129800481(-2)$ \\
\hline $1.326557084(0)$ & $2.062921869(-1)$ & $2.952229942(0)$ & $8.472947830(-4)$ \\
\hline $2.025948016(0)$ & $4.888991003(-2)$ & $\begin{array}{l}3.566193911(0) \\
4.241747838(0)\end{array}$ & $2.435886651(-5)$ \\
\hline $2.783290100(0)$ & $2.686707671(-3)$ & $\begin{array}{l}4.241747838(0) \\
5.043005292(0)\end{array}$ & $\begin{array}{l}1.990945921(-7) \\
2.103717408(-10)\end{array}$ \\
\hline $68470847(0)$ & $1.937314075(-5)$ & \multirow{2}{*}{\multicolumn{2}{|c|}{$h=18$}} \\
\hline \multicolumn{2}{|c|}{$h=11$} & & \\
\hline $0.000000000(0)$ & $7.554914856(-2)$ & $\begin{array}{l}5.035201634(-1) \\
1.010368387(0)\end{array}$ & $\begin{array}{l}9.928562581(-2) \\
1.874603945(-1)\end{array}$ \\
\hline $9.042299282(-1)$ & $2.421818138(-1)$ & $1.524170619(0)$ & $\begin{array}{l}1.874003940(-1) \\
1.180374099(-1)\end{array}$ \\
\hline $1.572365710(0)$ & $1.404923383(-1)$ & $2.049231710(0)$ & $3.354805258(-2)$ \\
\hline $2.261858997(0)$ & $2.181658844(-2)$ & $2.591133790(0)$ & $4.504254444(-3)$ \\
\hline $3.007348751(0)$ & $8.438017624(-4)$ & $3.157848818(0)$ & $2.713303695(-4)$ \\
\hline $3.879417698(0)$ & $4.346193273(-6)$ & $3.762187352(0)$ & $6.352683435(-6)$ \\
\hline & $=12$ & $4.428532807(0)$ & $4.242154626(-8)$ \\
\hline $6.057638792(-1)$ & $1.547120485(-1)$ & $5.220271691(0)$ & $3.614323562(-11)$ \\
\hline $1.220055037(0)$ & $2.088760748(-1)$ & \multicolumn{2}{|c|}{$h=19$} \\
\hline $1.853107652(0)$ & $7.164293661(-2)$ & $0.000000000(0)$ & $3.592678346(-2)$ \\
\hline $2.519735686(0)$ & $7.666245512(-3)$ & $7.033431134(-1)$ & $1.563101704(-1)$ \\
\hline $3.246608978(0)$ & $2.153455980(-4)$ & $1.214092716(0)$ & $1.715435896(-1)$ \\
\hline $4.101337596(0)$ & $8.117349108(-7)$ & $1.724429453(0)$ & $7.810756957(-2)$ \\
\hline \multirow{2}{*}{\multicolumn{2}{|c|}{$h=13$}} & $2.244120841(0)$ & $1.723645659(-2)$ \\
\hline & & $2.780040191(0)$ & $1.858876950(-3)$ \\
\hline $0.000000000(0)$ & $6.043931840(-2)$ & $3.340557183(0)$ & $9.162629181(-5)$ \\
\hline $8.382134662(-1)$ & $2.158018231(-1)$ & $3.938573396(0)$ & $1.771792021(-6)$ \\
\hline $1.453070399(0)$ & $1.575363996(-1)$ & $4.598379340(0)$ & $9.778930133(-9)$ \\
\hline $2.078631473(0)$ & $3.663398365(-2)$ & $5.382950244(0)$ & $6.788678392(-12)$ \\
\hline $2.735881148(0)$ & $2.861008745(-3)$ & \multirow{2}{*}{\multicolumn{2}{|c|}{$h=20$}} \\
\hline $3.452675336(0)$ & $6.041745852(-5)$ & & \\
\hline $4.296424463(0)$ & $1.709769798(-7)$ & $4.794507071(-1)$ & $8.773540673(-2)$ \\
\hline & $=14$ & $\begin{array}{l}9.614996344(-1) \\
1.448934251(0)\end{array}$ & $1.263417796(-1)$ \\
\hline $5.650695832(-1)$ & $1.315622572(-1)$ & $1.944962949(0)$ & $4.317805136(-2)$ \\
\hline $1.136115585(0)$ & $2.045709348(-1)$ & $2.453552124(0)$ & $7.554889021(-3)$ \\
\hline $1.719992575(0)$ & $9.105886603(-2)$ & $2.979991208(0)$ & $6.641078139(-4)$ \\
\hline $2.325732486(0)$ & $1.502666215(-2)$ & $3.531972877(0)$ & $2.709390011(-5)$ \\
\hline $2.967166928(0)$ & $8.804470588(-4)$ & $4.121995548(0)$ & $4.368737935(-7)$ \\
\hline $3.669950373(0)$ & $1.426473561(-5)$ & $4.773992343(0)$ & $2.009849940(-9)$ \\
\hline $4.499990707(0)$ & $3.083000771(-8)$ & $5.550351873(0)$ & $1.146110776(-12)$ \\
\hline
\end{tabular}


Table 4. (Continued)

\begin{tabular}{|c|c|c|c|}
\hline & & & \\
\hline$r_{i}$ & $B_{i}$ & $r_{i}$ & $B_{i}$ \\
\hline & $=2$ & & 13 \\
\hline $1.414213562(0)$ & $5.000000000(-1)$ & $0.000000000(0)$ & $3.571428613(-2)$ \\
\hline & $=3$ & $9.433668510(-1)$ & $2.159434172(-1)$ \\
\hline $0.000000000(0)$ & $3.333333333(-1)$ & $\begin{array}{l}1.559853914(0) \\
2.183163663(0)\end{array}$ & $\begin{array}{l}2.049051304(-1) \\
5.623828175(-2)\end{array}$ \\
\hline $1.732050808(0)$ & $3.333333333(-1)$ & $2.836944615(0)$ & $4.941414537(-3)$ \\
\hline & $=4$ & $3.549706167(0)$ & $1.142640717(-4)$ \\
\hline $1.126032501(0)$ & $3.943375673(-1)$ & $4.388854356(0)$ & $3.490093630(-7)$ \\
\hline $2.175327747(0)$ & $1.056624327(-1)$ & & 14 \\
\hline & $=5$ & $6.789876433(-1)$ & $1.131052983(-1)$ \\
\hline $0.000000000(0)$ & $1.666666667(-1)$ & $1.250434400(0)$ & $2.348543538(-1)$ \\
\hline $1.414213562(0)$ & $3.750000000(-1)$ & $1.830860591(0)$ & $1.265612582(-1)$ \\
\hline $2.449489743(0)$ & $4.166666667(-2)$ & $2.432343982(0)$ & 2. $390155444(-2)$ \\
\hline & $=6$ & $\begin{array}{l}3.069315784(0) \\
3.767514505(0)\end{array}$ & $\begin{array}{l}1.550229878(-3) \\
2.724233444(-5)\end{array}$ \\
\hline $9.673790506(-1)$ & $2.943407405(-1)$ & $4.592622008(0)$ & $6.316699406(-8)$ \\
\hline $\begin{array}{l}1.818077911(0) \\
2.785456961(0)\end{array}$ & $\begin{array}{l}1.956080296(-1) \\
1.005122987(-2)\end{array}$ & & 15 \\
\hline & & $0.000000000(0)$ & $2.777777758(-2)$ \\
\hline & & $8.849271664(-1)$ & $1.856328954(-1)$ \\
\hline $0.000000000(0)$ & $9.999999998(-2)$ & $1.460218218(0)$ & $2.111744224(-1)$ \\
\hline $1.231822666(0)$ & $3.418689189(-1)$ & $2.036753451(0)$ & $7.785882427(-2)$ \\
\hline $2.076435199(0)$ & $1.050368248(-1)$ & $2.632069602(0)$ & $1.089411340(-2)$ \\
\hline $3.028370814(0)$ & $3.094256323(-3)$ & $3.261980449(0)$ & $5.433840799(-4)$ \\
\hline & $=8$ & $3.952562204(0)$ & $7.458272172(-6)$ \\
\hline $8.621437977(-1)$ & $2.234352966(-1)$ & $4.769197755(0)$ & $1.340975865(-8)$ \\
\hline $1.603631818(0)$ & $2.388178862(-1)$ & & 16 \\
\hline $\begin{array}{l}2.393988043(0) \\
3.309666798(0)\end{array}$ & $\begin{array}{l}3.708889237(-2) \\
6.579248431(-4)\end{array}$ & $6.398308942(-1)$ & $9381627035(-2)$ \\
\hline & & $1.176844588(0)$ & $2.194926808(-1)$ \\
\hline & $=9$ & $1.719376211(0)$ & $1.449980353(-1)$ \\
\hline $0.000000000(0)$ & $6.666666667(-2)$ & $2.276388170(0)$ & $3.757069232(-2)$ \\
\hline $1.107593456(0)$ & $2.957070118(-1)$ & $2.856870611(0)$ & $3.966323324(-3)$ \\
\hline $1.847297312(0)$ & $1.558127471(-1)$ & $3.474198487(0)$ & $1.543210684(-4)$ \\
\hline $2.627297586(0)$ & $1.497213789(-2)$ & $4.153280092(0)$ & $1.674479104(-6)$ \\
\hline & $1.747698844(-4)$ & $4.958422657(0)$ & $2.360696415(-9)$ \\
\hline & $=10$ & & \\
\hline $7.855131146(-1)$ & $1.740072700(-1)$ & & \\
\hline $1.453604471(0)$ & $2.511403371(-1)$ & $0.000000000(0)$ & $2.222222281(-2)$ \\
\hline $2.147285065(0)$ & $7.045795974(-2)$ & $8.362597636(-1)$ & 1. $607235676(-1)$ \\
\hline $8114382(0)$ & $4.359946513(-3)$ & $1.377975506(0)$ & $2.105340253(-1)$ \\
\hline $3.776255164(0)$ & $3.448666179(-5)$ & $1.917710815(0)$ & $9.702900570(-2)$ \\
\hline & $=11$ & $3.044244703(0)$ & $1.571946046(-3)$ \\
\hline $0.000000000(0)$ & $0(-2)$ & $3.655234366(0)$ & $4.893816948(-5)$ \\
\hline $1.015435446(0)$ & $128(-1)$ & $4.327601954(0)$ & $4.277503020(-7)$ \\
\hline $1.684402809(0)$ & $1.879848286(-1)$ & $5.125294239(0)$ & $4.802950017(-10)$ \\
\hline $2.370715983(0)$ & $3.412185422(-2)$ & & \\
\hline $3.111737431(0)$ & $1.474981901(-3)$ & & 18 \\
\hline $3.978501467(0)$ & $8.298740836(-6)$ & $6.067771032(-1)$ & $7.901546073(-2)$ \\
\hline & $=12$ & $1.115059623(0)$ & $\begin{array}{l}2.032912912(-1) \\
1.574912414(-1)\end{array}$ \\
\hline $7.264076828(-1)$ & $1.388250710(-1)$ & $2.148693211(0)$ & $5.188776005(-2)$ \\
\hline $1.340261098(0)$ & $2.469552915(-1)$ & $2.687338189(0)$ & $7.788818857(-3)$ \\
\hline $1.968918871(0)$ & $1.015021484(-1)$ & $3.250741578(0)$ & $5.124359851(-4)$ \\
\hline $2.630364341(0)$ & $1.233441018(-2)$ & $3.851741751(0)$ & $1.290010569(-5)$ \\
\hline $3.351807039(0)$ & $3.815213838(-4)$ & $4.514662998(0)$ & $9.167797754(-8)$ \\
\hline $4.200709887(0)$ & $1.557519694(-6)$ & $5.302673230(0)$ & $8.271647273(-11)$ \\
\hline
\end{tabular}


TABle 5 .

Quadrature Formulas for the Integral $\int_{-\infty}^{\infty}|r|^{n-1} e^{-|r|} f(r) d r$

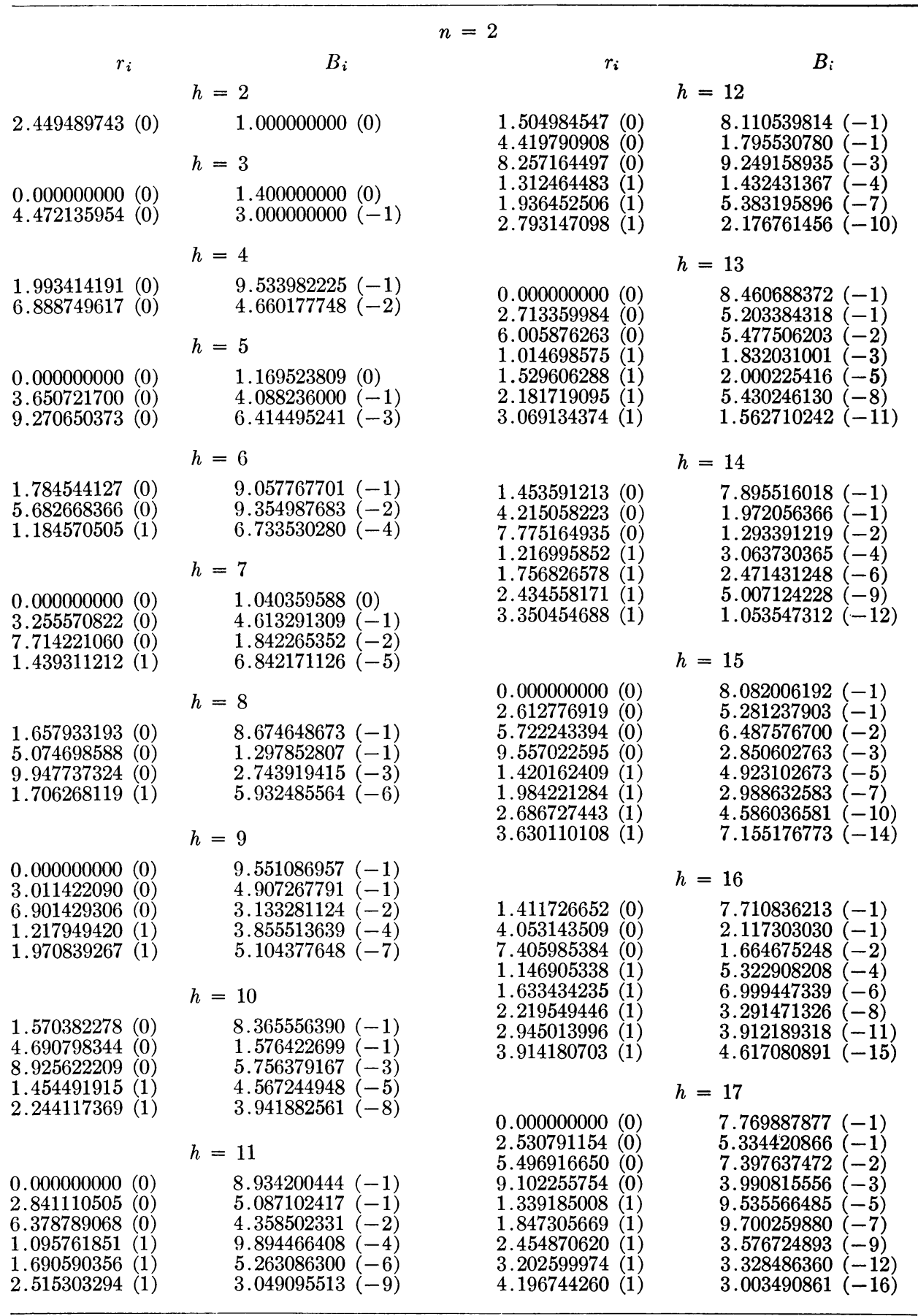


TABLe 5. (Continued)

\begin{tabular}{|c|c|c|c|}
\hline \multicolumn{4}{|c|}{$n=2$} \\
\hline$r_{i}$ & $B_{i}$ & $r_{i}$ & $B_{i}$ \\
\hline \multicolumn{2}{|c|}{$h=18$} & & $\begin{array}{l}=20 \\
7407489916(-1)\end{array}$ \\
\hline $1.376700177(0)$ & $7.549769410(-1)$ & $3.809845727(0)$ & $\begin{array}{l}7.407489916(-1) \\
2.342414464(-1)\end{array}$ \\
\hline $3.920785836(0)$ & $2.238963401(-1)$ & $6.868736260(0)$ & $2.383988972(-2)$ \\
\hline $7.111262558(0)$ & $2.029783818(-2)$ & 1.048743827 (1) & $1.141743053(-3)$ \\
\hline 1.092541981 (1) & $8.136253812(-4)$ & $1.469354218(1)$ & $2.759942059(-5)$ \\
\hline $1.541470582(1)$ & $1.513270088(-5)$ & $1.955773555(1)$ & $3.280384616(-7)$ \\
\hline 2.069231431 (1) & $1.222929718(-7)$ & 2.520496216 (1) & $1.739658808(-9)$ \\
\hline 2.696668115 (1) & $3.606728420(-10)$ & 3.185430024 (1) & $3.390285411(-12)$ \\
\hline 3.465275661 (1) & $2.671714183(-13)$ & 3.993538822 (1) & $1.638563875(-15)$ \\
\hline 4.483053563 (1) & $1.871902732(-17)$ & $5.056155489(1)$ & $7.127466841(-20)$ \\
\hline \multirow{2}{*}{\multicolumn{2}{|c|}{$h=19$}} & \multicolumn{2}{|c|}{$h=21$} \\
\hline & & $0.000000000(0)$ & $7.280504948(-1)$ \\
\hline $0.000000000(0)$ & $7.506624325(-1)$ & $2.403657913(0)$ & $5.396115951(-1)$ \\
\hline $2.462203560(0)$ & $5.371033116(-1)$ & $5.156977216(0)$ & $8.963950070(-2)$ \\
\hline $5.312154206(0)$ & $8.219337154(-2)$ & $8.436448890(0)$ & $6.479032616(-3)$ \\
\hline $8.737543593(0)$ & $5.210682402(-3)$ & $1.224931840(1)$ & $2.399266876(-4)$ \\
\hline $1.276024640(1)$ & $1.590671826(-4)$ & $1.663670269(1)$ & $4.652133232(-6)$ \\
\hline $1.744596401(1)$ & $2.335829865(-6)$ & 2.167605055 (1) & $4.516562638(-8)$ \\
\hline 2.291548468 (1) & $1.515308135(-8)$ & $2.749703529(1)$ & $1.972788939(-10)$ \\
\hline 2.938338728 (1) & $3.606268160(-11)$ & 3.432342199 (1) & $3.169555499(-13)$ \\
\hline 3.727247156 (1) & $2.144027471(-14)$ & $4.259167283(1)$ & 1. $253484080(-16)$ \\
\hline $4.767991124(1)$ & $1.176358097(-18)$ & $5.343096398(1)$ & $4.352749229(-21)$ \\
\hline \multicolumn{4}{|c|}{$n=3$} \\
\hline \multicolumn{2}{|c|}{$h=2$} & \multicolumn{2}{|c|}{$h=10$} \\
\hline \multirow[t]{2}{*}{$3.464101616(0)$} & $2.000000000(0)$ & \multirow{4}{*}{$\begin{array}{l}2.204007789(0) \\
5.534344976(0) \\
9.942229405(0) \\
1.572747040(1) \\
2.380096135(1)\end{array}$} & $1.510927918(0)$ \\
\hline & $=3$ & & $\begin{array}{l}4.649691274(-1) \\
2.385849965(-2)\end{array}$ \\
\hline $0.000000000(0)$ & & & $2.441943046(-4)$ \\
\hline $5.477225575(0)$ & $8.000000000(-1)$ & & $2.604962184(-7)$ \\
\hline \multicolumn{2}{|c|}{$h=4$} & \multicolumn{2}{|c|}{$h=11$} \\
\hline & $1.859072401(0)$ & \multirow{5}{*}{$\begin{array}{l}0.000000000(0) \\
3.485503203(0) \\
7.224827397(0) \\
1.197147010(1) \\
1.807971489(1) \\
2.649701152(1)\end{array}$} & \multirow{5}{*}{$\begin{array}{l}1.208885077(0) \\
1.230866895(0) \\
1.598170677(-1) \\
4.841270874(-3) \\
3.220522874(-5) \\
2.260192416(-8)\end{array}$} \\
\hline \multirow{2}{*}{$\begin{array}{l}2.820199646(0) \\
8.085778088(0)\end{array}$} & \multirow{2}{*}{$1.409275986(-1)$} & & \\
\hline & & & \\
\hline $0.000000000(0)$ & $1.831932773(0)$ & & \\
\hline $4.487554446(0)$ & $1.059643903(0)$ & & \\
\hline 1.044474147 (1) & $2.438971059(-2)$ & \multicolumn{2}{|c|}{$h=12$} \\
\hline \multicolumn{2}{|c|}{$h=6$} & \multirow{5}{*}{$\begin{array}{l}2.107862375(0) \\
5.212248703(0) \\
9.202027532(0) \\
1.421120388(1) \\
2.059284323(1) \\
2.931476633(1)\end{array}$} & $1.437279376(0)$ \\
\hline $2517630320(0)$ & $1.715600565(0)$ & & $5.238460417(-1)$ \\
\hline $6.699974358(0)$ & $2.815797622(-1)$ & & $3.810836809(-2)$ \\
\hline 1.312471408 (1) & $2.819672615(-3)$ & & $7.626909885(-4)$ \\
\hline & $=7$ & & $\begin{array}{l}3.521730919(-6) \\
1.706558796(-9)\end{array}$ \\
\hline $0.000000000(0)$ & $1.531269902(0)$ & & $=13$ \\
\hline $4.001421506(0)$ & $1.164873541(0)$ & $0.000000000(0)$ & $1.110152546(0)$ \\
\hline $8.725917405(0)$ & $6.915044224(-2)$ & $3.325514693(0)$ & $1.237261671(0)$ \\
\hline $1.565108384(1)$ & $3.410659140(-4)$ & $6.801535403(0)$ & $1.986398605(-1)$ \\
\hline & & 1.109179438 (1) & $8.900170086(-3)$ \\
\hline $2.332514115(0)$ & $1.601558198(0)$ & $\begin{array}{l}1.637867916(1) \\
2.303701983(1)\end{array}$ & $\begin{array}{l}1.216278155(-4) \\
3.974061224(-7)\end{array}$ \\
\hline $5.988121274(0)$ & $3.869802166(-1)$ & $3.206078045(1)$ & $1.350850632(-10)$ \\
\hline $\begin{array}{l}1.106562850(1) \\
1.839003601(1)\end{array}$ & $\begin{array}{l}1.142961744(-2) \\
3.196788260(-5)\end{array}$ & & $=14$ \\
\hline & & & \\
\hline & & $4.968086026(0)$ & $5.693437598(-1)$ \\
\hline $0.000000000(0)$ & $1.341423259(0)$ & $8.665734947(0)$ & $5.294734563(-2)$ \\
\hline $3.698153067(0)$ & $1.211157884(0)$ & 1.318598904 (1) & $1.624651243(-3)$ \\
\hline $7.815156311(0)$ & $1.162265803(-1)$ & 1.870620217 (1) & $1.609716258(-5)$ \\
\hline 1.328904863 (1) & $1.900745585(-3)$ & $2.560830442(1)$ & $3.879275669(-8)$ \\
\hline $2.101752165(1)$ & $3.160268315(-6)$ & $3.490578944(1)$ & $9.565745752(-12)$ \\
\hline
\end{tabular}


TABLE 5. (Continued)

$$
n=3
$$

$r_{i}$

$h=15$

$0.000000000(0)$

$3.199320722(0)$

$6.478534025(0)$

1.044888059 (1)

$1.521635732(1)$

2.097571715 (1)

2.812195646 (1)

3.769005592 (1)

$1.970691130(0)$

$4.774553568(0)$

8.253624968 (0)

1.243028441 (1)

1.740405855 (1)

2.337310892 (1)

3.073982925 (1)

4.055727188 (1)

0.000000000 (0)

$3.096345719(0)$

$6.221354460(0)$

9.951826534 (0)

1.435330447 (1)

1.954077575 (1)

2.572172581 (1)

3.330814667 (1)

4.337190490 (1)

$1.919192048(0)$

4.616108414 (0)

7.923860054 (0)

1. 184245453 (1)

1.643031385 (1)

2.180434951 (1)

2.817603515 (1)

3.596424118 (1)

4.625758865 (1)

$h=16$

$h=17$

$h=18$

$$
\begin{aligned}
& 1.033190220(0) \\
& 1.236526140(0) \\
& 2.328276642(-1) \\
& 1.375112000(-2) \\
& 2.977880891(-4) \\
& 2.173861565(-6) \\
& 3.910026664(-9) \\
& 7.071795600(-13)
\end{aligned}
$$

1.324194339 (0)

$6.052536059(-1)$

$6.769597291(-2)$

$2.810394009(-3)$

$4.543394453(-5)$

$2.537413815(-7)$

$3.505591573(-10)$

$4.767319036(-14)$

$9.711563020(-1)$

$1.231819983(0)$

$2.629025617(-1)$

$1.911837123(-2)$

$5.738780745(-4)$

$7.024148062(-6)$

$3.030226055(-8)$

$3.242045709(-11)$

$3.342430773(-15)$

$1.279510006(0)$

$6.341147499(-1)$

$8.200025545(-2)$

$4.276145443(-3)$

$9.790032305(-5)$

$9.396585962(-7)$

$3.213850652(-9)$

$2.719865679(-12)$

$2.166944216(-16)$

$n=4$

$h=2$

4.472135954 (0)

$6.000000000(0)$

$h=3$

$6.285714286(0)$

2.857142857 (0)

$h=4$

$3.650721700(0)$

$9.270650373(0)$

$0.000000000(0)$

5.330999200 (0)

1.160949816 (1)

$3.255570822(0)$

$7.714221060(0)$

1.439311212 (1)

5.448706474 (0)

$5.512935263(-1)$

$h=5$

$4.406015038(0)$

$3.683311857(0)$

$1.136806245(-1)$

$h=6$

4.889508150 (0)

$1.096317494(0)$

$r_{i}$

$0.000000000(0)$
$3.010143055(0)$
$6.010138717(0)$
$9.552340905(0)$
$1.367839955(1)$
$1.846110981(1)$
$2.402513440(1)$
$3.058816533(1)$
$3.857691826(1)$
$4.909704018(1)$

$1.875226838(0)$

4.483160491 (0)

$7.652027323(0)$

1.136784126 (1)

1.566480249 (1)

2.061689995 (1)

2.635140655 (1)

3.308973046 (1)

4.126492482 (1)

5.199827654 (1)

$0.000000000(0)$

$2.936536412(0)$

5.832543673 (0)

9.222009388 (0)

1.313137730 (1)

1.760850657 (1)

2.273431224 (1)

2.864088984 (1)

3.555437332 (1)

4.391462270 (1)

5.485863319 (1) $h=19$

$B_{i}$

$$
\begin{aligned}
& 9.198551077(-1) \\
& 1.224868813(0) \\
& 2.894400128(-1) \\
& 2.479411924(-2) \\
& 9.525272967(-4) \\
& 1.684609673(-5) \\
& 1.278349190(-7) \\
& 3.489146258(-10) \\
& 2.349920788(-13) \\
& 1.456108222(-17)
\end{aligned}
$$

$h=20$

$1.240486887(0)$
$6.576705943(-1)$
$9.568956071(-2)$
$5.972488440(-3)$
$1.779405062(-4)$
$2.513325777(-6)$
$1.544864552(-8)$
$3.430114664(-11)$
$1.868779188(-14)$
$9.145065859(-19)$

$h=21$

$8.765640821(-1)$

$1.216663536(0)$

$3.129627181(-1)$

$3.062830595(-2)$

$1.429596653(-3)$

$3.342033178(-5)$

$3.796867514(-7)$

$1.900657295(-9)$

$3.449437060(-12)$

$1.527425667(-15)$

$5.933632119(-20)$

$h=7$

$1.417435642(-2)$
$0.000000000(0)$

$4.755716499(0)$

$9.735614108(0)$

1.689931243 (1)

3.465648514 (0)

3.946919289 (0)

$1.983992062(-3)$

$$
h=8
$$

$3.011422090(0)$

$6.901429306(0)$

1.217949420 (1)

1.970839267 (1)

$4.450235787(0)$

$1.492373229(0)$

$5.719271981(-2)$

$1.982646151(-4)$

$h=9$

$0.000000000(0)$

$4.393542292(0)$

1.439445793 (1)

2.231769279 (1)
$3.182724615(-1)$

8.729737529 (0)
$2.897521330(0)$

$4.011677832(0)$

$5.285974076(-1)$

$1.094195263(-2)$

$2.214240146(-5)$ 
TABLE 5. (Continued)

\begin{tabular}{|c|c|c|c|}
\hline \multicolumn{4}{|c|}{$n=4$} \\
\hline$r_{i}$ & $h=10$ & \multicolumn{2}{|r|}{$h=17$} \\
\hline $2.841110505(0)$ & $4.106262729(0)$ & $0.000000000(0)$ & $1.862973318(0)$ \\
\hline $6.378789068(0)$ & $1.773428833(0)$ & $3.668871779(0)$ & $3.813238833(0)$ \\
\hline $1.095761851(1)$ & \multirow{2}{*}{$\begin{array}{l}1.188022678(-1) \\
1.504240460(-3)\end{array}$} & \multirow{2}{*}{$6.948595197(0)$} & $1.144406874(0)$ \\
\hline $1.690590356(1)$ & & & \\
\hline $2.515303294(1)$ & $1.929086705(-6)$ & \multirow{2}{*}{1.531451417 (1) } & $3.912057383(-3)$ \\
\hline \multicolumn{2}{|r|}{$h=11$} & & \multirow{4}{*}{$\begin{array}{l}5.644537982(-5) \\
2.806698694(-7) \\
3.412631516(-10) \\
3.980940502(-14)\end{array}$} \\
\hline $0.000000000(0)$ & \multirow{2}{*}{$\begin{array}{l}2.514931169(0) \\
3.996302185(0)\end{array}$} & 2.689191314 (1) & \\
\hline $4.138264916(0)$ & & $3.458604320(1)$ & \\
\hline $8.072947687(0)$ & $7.183484926(-1)$ & $4.477049252(1)$ & \\
\hline $1.298383257(1)$ & $2.766034904(-2)$ & & $h=18$ \\
\hline $1.924886297(1)$ & $2.232034015(-4)$ & $2.462203560(0)$ & $3.256160021(0)$ \\
\hline $2.783295386(1)$ & $1.856946795(-7)$ & $5.312154206(0)$ & $2.319413298(0)$ \\
\hline & $h=12$ & $8.737543593(0)$ & $3.978078183(-1)$ \\
\hline $2.713359984(0)$ & $3.830899293(0)$ & $1.276024640(1)$ & $2.589993713(-2)$ \\
\hline $6.005876263(0)$ & $1.975766597(0)$ & $1.744596401(1)$ & $7.109370552(-4)$ \\
\hline $1.014698575(1)$ & $1.886283300(-1)$ & 2.291548468 (1) & $7.957177568(-6)$ \\
\hline 1.529606288 (1) & $4.679918195(-3)$ & $2.938338728(1)$ & $3.113592239(-8)$ \\
\hline $2.181719095(1)$ & $2.584741882(-5)$ & $3.727247156(1)$ & $2.978562584(-11)$ \\
\hline 3.069134374 (1) & $1.472008322(-8)$ & $4.767991124(1)$ & $2.674301836(-15)$ \\
\hline & $h=13$ & & $h=19$ \\
\hline $0.000000000(0)$ & $2.238428450(0)$ & $0.000000000(0)$ & $1.728866368(0)$ \\
\hline $3.945591725(0)$ & $3.946646291(0)$ & $3.564606385(0)$ & $3.743204656(0)$ \\
\hline $7.599799179(0)$ & $8.828157687(-1)$ & $6.710834845(0)$ & $1.248087959(0)$ \\
\hline $1.203650356(1)$ & $5.048268586(-2)$ & $1.036840210(1)$ & $1.376778296(-1)$ \\
\hline $1.745891997(1)$ & $8.378015939(-4)$ & $1.459676318(1)$ & $6.460355281(-3)$ \\
\hline 2.425218757 (1) & $3.226547215(-6)$ & $1.947546116(1)$ & $1.348331481(-4)$ \\
\hline $3.342302084(1)$ & $1.275006919(-9)$ & 2.513295296 (1) & $1.179254048(-6)$ \\
\hline & $h=14$ & 3.179001649 (1) & $3.649770083(-9)$ \\
\hline & & $3.987725284(1)$ & $2.759290090(-12)$ \\
\hline $\begin{array}{l}2.612776919(0) \\
5.722243394(0)\end{array}$ & $\begin{array}{l}3.605291571(0) \\
2.124296621(0)\end{array}$ & $5.050869597(1)$ & $1.915768270(-16)$ \\
\hline $9.557022595(0)$ & $2.603645948(-1)$ & & $h=20$ \\
\hline $1.420162409(1)$ & $9.929215102(-3)$ & & \\
\hline 1.984221284 (1) & $1.176664727(-4)$ & $2.403657913(0)$ & $3.117644499(0)$ \\
\hline $2.686727443(1)$ & $3.310432502(-7)$ & $5.156977216(0)$ & $2.383909993(0)$ \\
\hline $3.630110108(1)$ & $9.428876866(-11)$ & $8.436448890(0)$ & $4.611365285(-1)$ \\
\hline & & $1.224931840(1)$ & $3.599999209(-2)$ \\
\hline & $h=15$ & $1.663670269(1)$ & $1.287616861(-3)$ \\
\hline & $2.028430719(0)$ & $2.167605055(1)$ & $2.122112229(-5)$ \\
\hline $3.793305759(0)$ & $3.882546844(0)$ & 2.749703529 (1) & $1.491599971(-7)$ \\
\hline $7.237614603(0)$ & $1.023742824(0)$ & 3.432342199 (1) & $3.734044767(-10)$ \\
\hline $1.134139790(1)$ & $7.743696134(-2)$ & 4.259167283 & $2.273883541(-13)$ \\
\hline $1.623004363(1)$ & $2.040427395(-3)$ & $5.343096398(1)$ & $1.242652410(-17)$ \\
\hline $2.210650396(1)$ & $1.754643554(-5)$ & & \\
\hline $2.937215978(1)$ & $3.643463895(-8)$ & & $h=21$ \\
\hline $3.907253683(1)$ & $7.546144982(-12)$ & $0.000000000(0)$ & $1.617675191(0)$ \\
\hline & & $3.475483011(0)$ & $3.674629246(0)$ \\
\hline & $h=16$ & $6.510593006(0)$ & $1.337662369(0)$ \\
\hline & $3.416645282(0)$ & $1.000875214(1)$ & $1.689521362(-1)$ \\
\hline $5.496916650(0)$ & $2.235276993(0)$ & 1.401368507 (1) & $9.648631588(-3)$ \\
\hline $9.102255754(0)$ & $3.306432984(-1)$ & $1.857966859(1)$ & $2.665099398(-4)$ \\
\hline 1.339185008 (1) & $1.710124214(-2)$ & $2.379102183(1)$ & $3.491654726(-6)$ \\
\hline 1.847305669 (1) & $3.310250774(-4)$ & 2.978225375 (1) & $1.980969064(-8)$ \\
\hline $2.454870620(1)$ & $2.155473827(-6)$ & 3.678186061 (1) & $4.025361928(-11)$ \\
\hline 3.202599974 (1) & $3.413910828(-9)$ & 4.523307680 (1) & $1.981137602(-14)$ \\
\hline 4.196744260 (1) & $5.289947051(-13)$ & $5.628064129(1)$ & $8.553609979(-19)$ \\
\hline
\end{tabular}


witz and Weiss [12]. In these cases our values of $x_{k}^{(\alpha)}$ agreed exactly with theirs to $10 \mathrm{~S}$ but a few values of the $A_{k}{ }^{(\alpha)}$ differed in the 10-th figure.

5. Examples of Spherical Product Formulas. In this section we explain in greater detail for $n=3$ how the spherical product formulas which we discussed in Section 3 are constructed.

We can write the points in a formula of degree $2 h-1$, let us say for the integral $U$, as

$$
\left(\lambda_{i, j, k}, \mu_{i, j, k}, \nu_{i, j, k}\right)
$$

where

$$
\begin{aligned}
\lambda_{i, j, k} & =r_{i} \cos \theta_{2, j} \cos \theta_{1, k} \\
\mu_{i, j, k} & =r_{i} \cos \theta_{2, j} \sin \theta_{1, k} \\
\nu_{i, j, k} & =r_{i} \sin \theta_{2, j}
\end{aligned}
$$

and where the subscripts $i, j, k$ range independently over the integers $1,2, \cdots, h$. Since we are considering a formula for $U$ the $r_{i}$ are the $h$ points in the formula (3.8):

$$
\int_{-\infty}^{\infty} r^{2} e^{-r^{2}} r^{\alpha} d r=\sum_{i=1}^{h} B_{i} r_{i}^{\alpha}, \quad \alpha=0,1, \cdots, 2 h-1
$$

The values of $\sin \theta_{1, k}$ are the $h$ roots of the Chebyshev polynomial of degree $h$ of the first kind. These values of $\sin \theta_{1, k}$ are symmetrically located on the interval $[-1,+1]$ and the corresponding values of $\cos \theta_{1, k}$ are the positive values of $\sqrt{1-\sin ^{2} \theta_{1, k}}$. Similarly, the $\sin \theta_{2, j}$ are the $h$ roots of the Legendre polynomial of degree $h$ and are also symmetrically located on $[-1,+1]$ and the corresponding values of $\cos \theta_{2, j}$ are the positive values of $\sqrt{1-\sin ^{2} \theta_{2, j}}$. The coefficients corresponding to $(5.1)$ are

$$
A_{i, j, k}=B_{i} B_{2, j} B_{1, k}
$$

where $B_{i}$ is the coefficient in $(3.8) ; B_{1, k}=\pi / h$ is the coefficient in (3.7); and the $B_{2, j}$ are the Gauss-Legendre coefficients.

Taking $h=4$ we obtain a formula of degree 7 which, for $n=3$, contains 64 points. The one-dimensional formulas from which this 64 point formula is constructed are, rounded to 4 decimals:

$$
\begin{array}{rcrcrc}
r_{i} & B_{i} & \sin \theta_{2, j} & \multicolumn{1}{c}{B_{2, j}} & \sin \theta_{1, k} & B_{1, k} \\
-2.0202 & 0.0814 & -0.8611 & 0.3479 & -0.9239 & 0.7854 \\
-0.9586 & 0.3617 & -0.3400 & 0.6521 & -0.3827 & 0.7854 \\
0.9586 & 0.3617 & 0.3400 & 0.6521 & 0.3827 & 0.7854 \\
2.0202 & 0.0814 & 0.8611 & 0.3479 & 0.9239 & 0.7854
\end{array}
$$

The points (5.1) and coefficients (5.2) are easily calculated on a computer. Suppose we wish to use the above 64 point formula for the evaluation of

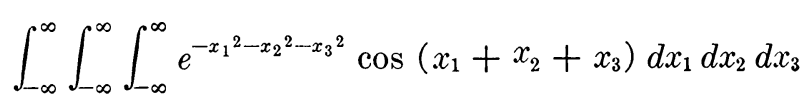


A complete Fortran program to calculate the points and to evaluate this integral is:

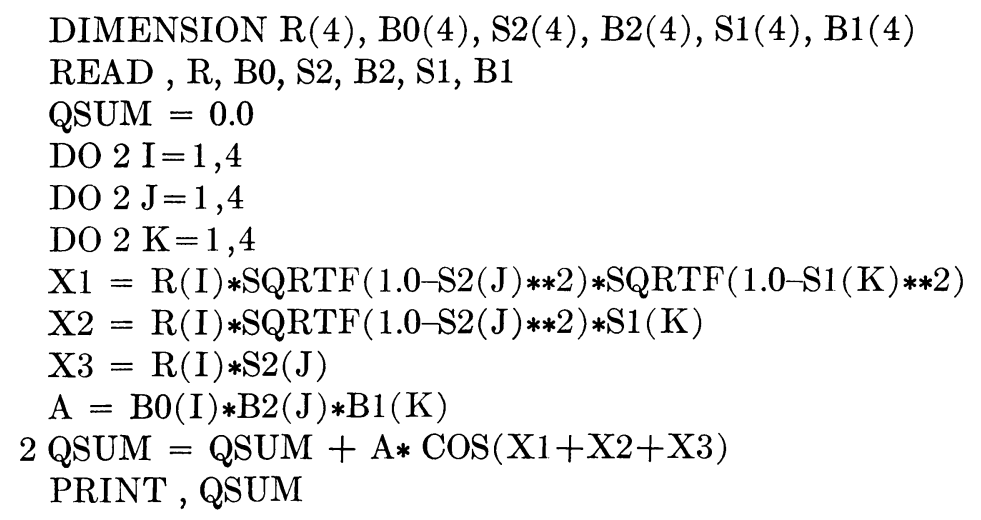

If $h$ is odd the points (5.1) and coefficients (5.2) can be calculated in exactly the same way as for even $h$. For example, let us take $h=5$. The resulting formula of degree 9 uses $5^{3}-5^{2}+1=101$ points. We can consider this formula to consist of $5^{3}=125$ points, but since $r_{3}=0,25$ of the points coincide with the origin. The coefficient for the point at the origin is then

$$
A_{3, j, k}=B_{3} \sum_{j, k=1}^{5} B_{2, j} B_{1, k} .
$$

The above program can still be used if we replace the 4-dimensional arrays by the appropriate 5-dimensional arrays.

Table 6 compares the exact value of (5.3) with the above 64 point and 101 point formulas and also with each of the formulas for 3 dimensions of Table 1.

6. Approximate Integration Formulas and Orthogonal Polynomials. For a single variable it is well known that a necessary and sufficient condition that the

\begin{tabular}{lc}
\multicolumn{2}{c}{ TABLE 6} \\
Values of $(5.3)$ \\
\hline Exact value & 2.630292 \\
Formula I & 2.325022 \\
Formula II & 1.888699 \\
Formula III & 2.446723 \\
Formula IV & 2.731897 \\
Formula VII & 2.731446 \\
Formula VIII & 2.671828 \\
& $(2.338437)$ \\
Formula IX & 2.691434 \\
Formula X & 2.699224 \\
& $(2.637765)$ \\
Formula XI & 2.655450 \\
& $(2.640705)$ \\
64 points & 2.623610 \\
101 points & 2.630861 \\
\hline
\end{tabular}


$N$ points $\nu_{1}, \nu_{2}, \cdots, \nu_{N}$ can be used to construct a quadrature formula of degree $k$ for

$$
\int_{R} w(x) f(x) d x
$$

where $w(x)$ is non-negative in $R$, is that the polynomial

$$
P_{N}(x)=\left(x-\nu_{1}\right)\left(x-\nu_{2}\right) \cdots\left(x-\nu_{N}\right)
$$

be orthogonal over $R$ with respect to $w(x)$ to all polynomials of degree $\leqq k-N$, that is

$$
\int_{R} w(x) P_{N}(x) x^{i} d x=0, \quad i=0,1, \cdots, k-N
$$

(see, for example, Krylov [9], p. 96). Thus, any $N$ points can be used to construct a quadrature formula of degree $N-1$. If $P_{N}(x)$ is orthogonal to the constant:

$$
\int_{R} w(x) P_{N}(x) d x=0
$$

then a quadrature formula using the $\nu_{i}$ can be found which has degree $N$. If $P_{N}(x)$ is orthogonal to all polynomials of degree $<N$ then the formula can be constructed to have degree $2 N-1$ and this is the highest degree which can be obtained using $N$ points.

It seems likely that approximate integration formulas for functions of $n$ variables are also closely related to orthogonal polynomials in $n$ variables, but the exact relationship is not known. Thus, one would expect that the $N$ points $\nu_{i}$ $(i=1, \cdots, N)$ in an integration formula (1.1) of degree $k$ would be the common solutions of a system of $n$ polynomials

$$
P_{h_{i}}^{i}\left(x_{1}, \cdots, x_{n}\right)=0, \quad i=1,2, \cdots, n
$$

where $P_{h_{i}}^{i}$ has degree $h_{i}$ and is orthogonal to all polynomials of degree $\leqq k-h_{\imath}$ :

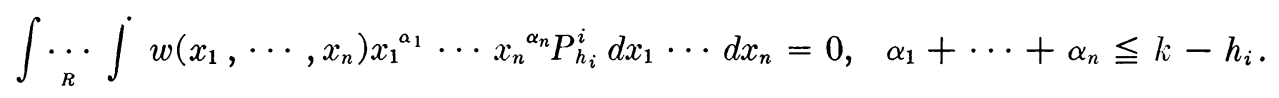

If $Q_{h}{ }^{1}$ and $Q_{h}{ }^{2}$ are two linearly independent polynomials of degree $h$ in $x_{1}, \cdots, x_{n}$, each of which is orthogonal over $R$ with respect to $w\left(x_{1}, \cdots, x_{n}\right)$ to all polynomials of degree $<h$ then any linear combination

$$
a Q_{h}{ }^{1}+b Q_{h}{ }^{2}
$$

will also be orthogonal to all polynomials of degree $<h$.

If $w\left(x_{1}, \cdots, x_{n}\right)$ is positive throughout $R$ it is then easy to show that there are exactly $(n+h-1) ! /(n-1) ! h !-$ which is the number of monomials of degree $h$-linearly independent polynomials of degree $h$ which are orthogonal to all polynomials of lower degree (Jackson [8] gives the proof for $n=2$ ). There will usually be no orthogonality relationship between polynomials of the same degree.

A complete system of polynomials which are orthogonal to all polynomials of lower degree for the integral $U$ is the system of generalized Hermite polynomials 
which have been studied by Appell and Kampé de Fériet [1]. These are the polynomials

$$
\begin{aligned}
H_{\alpha_{1}, \cdots, \alpha_{n}} & =(-1)^{\alpha_{1}+\cdots+\alpha_{n}} e^{x_{1}{ }^{2}+\cdots+x_{n}{ }^{2}} \frac{\partial^{\alpha_{1}+\cdots+\alpha_{n}}}{\partial x_{1}^{\alpha_{1}} \cdots \partial{x_{n}{ }^{\alpha_{n}}}^{\alpha_{1}}}\left[e^{-x_{1}{ }^{2}-\cdots-x_{n}{ }^{2}}\right] \\
& =H_{\alpha_{1}}(x) \cdots H_{\alpha_{n}}\left(x_{n}\right)
\end{aligned}
$$

where $H_{\alpha}(x)$ is the Hermite polynomial of degree $\alpha$

$$
H_{\alpha}=(-1)^{\alpha} e^{x^{2}} \frac{d^{\alpha}}{d x^{\alpha}} e^{-x^{2}}
$$

which satisfies

$$
\int_{-\infty}^{\infty} e^{-x^{2}} H_{\alpha}(x) x^{i} d x=0, \quad i=0,1, \cdots, \alpha-1 .
$$

Orthogonal polynomials for the integral $V$ have not previously been studied. In Table 7 we give for $V$ a complete system of polynomials $G_{\alpha_{1}, \cdots, \alpha_{n}}$ of degree $\leqq \tilde{j}$ each polynomial being orthogonal to all polynomials of lower degree. The coefficients of each polynomial were determined by solving a linear system of equations.

If the $n$ polynomials (6.1) are all of degree $h$ and each is orthogonal to all polynomials of degree $<h$ one might expect that if these polynomials have a finite number of common solutions then these points could be used to form a quadrature formula of degree $2 h-1$. In the following section we show that this is not true:

TABLE 7

Orthogonal Polynomials for the Integral $V$.

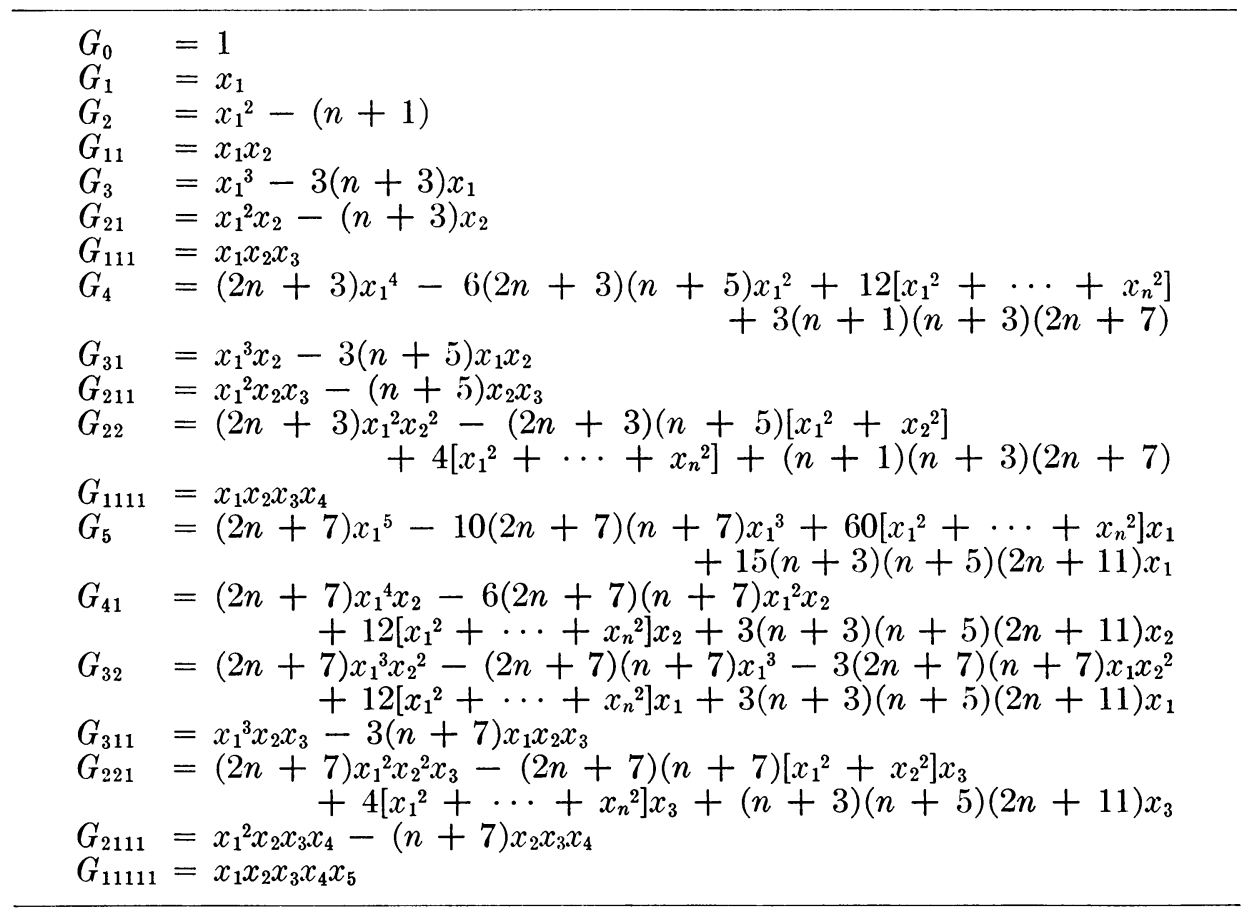


in some cases there are systems of polynomials, where each polynomial has degree $h$ and is orthogonal to all polynomials of degree $<h$, for which the common solutions cannot serve as the points in an integration formula of degree $2 h-1$.

If we know that an integration formula of a certain degree exists it is not known whether the points in such a formula must necessarily satisfy a system of equations of the form (6.1), however, no formula is known which does not have this property. In Section 8 we give systems of orthogonal polynomials which intersect in the points in the spherical product formulas discussed in Section 3. Given these polynomials it is easy to see how, in the particular cases of formulas for $U$ and $V$, they may be written as linear combinations of the polynomials $H_{\alpha_{1}, \cdots, \alpha_{n}}$ and $G_{\alpha_{1}, \cdots, \alpha_{n}}$ respectively.

7. Certain Orthogonal Polynomials from Which Approximate Integration Formulas Cannot Be Derived. In Section 6 we discussed certain questions about the relationship between approximate integration formulas and orthogonal polynomials. In this section we show that for Cartesian product regions there are sets of orthogonal polynomials, all polynomials of a set having degree $k$, whose common roots cannot be taken as the points in a quadrature formula of degree $2 k-1$.

Before we discuss the general case let us consider as an example the Hermite polynomials

$$
\begin{aligned}
H_{3,0}=8 x^{3}-12 x & H_{1,2}=8 x y^{2}-4 x \\
H_{2,1}=8 x^{2} y-4 y & H_{0,3}=8 y^{3}-12 y .
\end{aligned}
$$

Each of these polynomials satisfies

$$
\int_{-\infty}^{\infty} \int_{-\infty}^{\infty} e^{-x^{2}-y^{2}} H_{\alpha, \beta}(x, y) x^{\gamma} y^{\delta} d x d y=0 \quad \begin{aligned}
& \alpha+\beta=3 \\
& \gamma+\delta<3
\end{aligned}
$$

The nine common solutions of

$$
\begin{aligned}
& H_{3,0}=0 \\
& H_{0.3}=0
\end{aligned}
$$

give the Cartesian product formula of degree 5 . On the other hand, the common solutions of none of the other pairs of (7.1) can be taken as the points in a quadrature formula of degree 5 . The two pairs which have a finite number of common solutions are:

$$
\begin{array}{rcc} 
& \begin{array}{c}
\text { Solutions } \\
H_{3,0}=0 \\
H_{2,1}=0
\end{array} \\
& (0,0) \quad\left( \pm \sqrt{\frac{3}{2}}, 0\right) \\
H_{2,1}=0 & (0,0) \quad\left( \pm \sqrt{\frac{1}{2}}, \pm \sqrt{\frac{1}{2}}\right) .
\end{array}
$$

The pair

$$
\begin{aligned}
& H_{3,0}=0 \\
& H_{1,2}=0
\end{aligned}
$$


does not have a finite number of solutions; the solutions are seen to be the line $x=0$ and the points $\left( \pm \sqrt{\frac{3}{2}}, \pm \sqrt{\frac{1}{2}}\right)$. We will not consider this case further.

It is quite clear that the solutions of (7.2) cannot be used as the points in a quadrature formula of degree 5 since it will be impossible to make the formula exact for the six monomials $1, y, y^{2}, y^{3}, y^{4}, y^{5}$. The same is true for (7.3). In this case there are 3 distinct values of $y_{i}: 0, \sqrt{\frac{1}{2}},-\sqrt{\frac{1}{2}}$. In order that these $y_{i}$ may be used to exactly integrate the monomials $y^{k} \quad(k=0,1, \cdots, 5)$ it is necessary and sufficient that they be the roots of the cubic polynomial $H_{3}(y)=8 y^{3}-12 y$ which satisfies:

$$
\int_{-\infty}^{\infty} e^{-y^{2}} H_{3}(y) y^{\alpha} d y=0, \quad \alpha=0,1,2 .
$$

They are not the roots of this polynomial but are the combined roots of the two polynomials

$$
H_{1}(y)=2 y, \quad H_{2}(y)=4 y^{2}-2
$$

which then establishes our assertion.

This result may be extended to any Cartesian product region $R_{n}$ with a positive weight function, that is, to any region which satisfies

$$
\begin{aligned}
\int \underset{R_{n}}{\ldots} \int w\left(x_{1}, \cdots, x_{n}\right) x_{1}^{\alpha_{1}} \cdots x_{n}{ }^{\alpha_{n}} d x_{1} \cdots & d x_{n} \\
& =\int_{S^{1}} w_{1}\left(x_{1}\right) x_{1}{ }^{\alpha_{1}} d x_{1} \cdots \int_{S^{n}} w_{n}\left(x_{n}\right) x_{n}{ }^{\alpha_{n}} d x_{n}
\end{aligned}
$$

where the $S^{i}$ are assumed to be arbitrary regions on the lines $-\infty<x_{i}<\infty$ and each $w_{i}\left(x_{i}\right)$ is assumed non-negative throughout $S^{i}$. Let $P_{m}{ }^{i}\left(x_{i}\right), \quad m=0,1,2, \cdots$, $i=1, \cdots, n$, be the sequences of orthogonal polynomials which satisfy

$$
\int_{S^{i}} w_{i}\left(x_{i}\right) P_{m}^{i}\left(x_{i}\right) P_{n}^{i}\left(x_{i}\right) d x_{i}=0, \quad n \neq m \text {. }
$$

(The $P_{m}{ }^{i}\left(x_{i}\right)$ are unique up to a normalizing factor.) Then the polynomials

$$
P_{\alpha_{1}, \alpha_{2}, \cdots, \alpha_{n}}=P_{\alpha_{1}}^{1}\left(x_{1}\right) P_{\alpha_{2}}^{2}\left(x_{2}\right) \cdots P_{\alpha_{n}}^{n}\left(x_{n}\right)
$$

form an orthogonal system for the region $R_{n}$; that is,

$$
\int \ldots_{R_{n}} \int w\left(x_{1}, \cdots, x_{n}\right) P_{\alpha_{1}}, \cdots{ }_{\alpha_{n}} P_{\beta_{1}}, \cdots, \beta_{n} d x_{1} \cdots d x_{n}=0
$$

for $\alpha_{1}+\cdots+\alpha_{n} \neq \beta_{1}+\cdots+\beta_{n}$. This follows immediately from the fact that

$$
\int \ldots_{R_{n}} \int w\left(x_{1}, \cdots, x_{n}\right) x_{1}^{\beta_{1}} \cdots x_{n}^{\beta_{n}} P_{\alpha_{1}}, \ldots, \alpha_{n} d x_{1} \cdots d x_{n}=0
$$

whenever $\beta_{1}+\cdots+\beta_{n}<\alpha_{1}+\cdots+\alpha_{n}$. This is true since for at least one value of $i$ it must be true that $\beta_{i}<\alpha_{i}$ and for such an $i$

$$
\int_{S^{i}} w_{\imath}\left(x_{i}\right) x_{i}^{\beta_{i}} P_{\alpha_{i}}^{i} d x_{i}=0 .
$$


We can now prove

THEOREM. The only system of $n$ equations of the form

$$
\begin{aligned}
P_{\alpha_{1}, \cdots, \alpha_{n}} & =0 \\
P_{\beta_{1}, \cdots, \beta_{n}} & =0 \\
\cdots & \\
P_{\gamma_{1}, \cdots, \gamma_{n}} & =0
\end{aligned}
$$

for $\alpha_{1}+\cdots+\alpha_{n}=\beta_{1}+\cdots+\beta_{n}=\cdots=\gamma_{1}+\cdots+\gamma_{n}=k$, whose common solutions can be used as the points in an approximate integration formula of degree. $2 k-1$ for $R_{n}$ is the system

$$
\begin{aligned}
P_{k, 0, \cdots, 0} & =0 \\
P_{0, k, \cdots, 0} & =0 \\
\cdots & \\
P_{0,0, \cdots, k} & =0
\end{aligned}
$$

which gives the Cartesian product formula for $R_{n}$.

Proof. It is not difficult to prove that the solutions of the equations (7.5) give a Cartesian product formula of degree $2 k-1$ for $R_{n}$ (see, for example, Hammer and Wymore [5]). For any other set of equations (7.4) we notice that the subscripts have the property that for some $i, 1 \leqq i \leqq n$,

$$
\alpha_{i}+\beta_{i}+\cdots+\gamma_{i}=h \leqq k
$$

and if $h=k$ at least two of the integers $\alpha_{i}, \beta_{i}, \cdots, \gamma_{i}$ are different from zero.

Let us assume that this is true for $i=n$ so that $\alpha_{n}+\cdots+\gamma_{n}=h \leqq k$ and if $h=k$ at least two of the $\alpha_{n}, \cdots, \gamma_{n}$ are not zero. Let $\xi_{j}$ be the distinct values of $x_{n}$ which occur in the common solutions of (7.4). The $\xi_{j}$ are the combined roots of the polynomials

$$
P_{\alpha_{n}}^{n}\left(x_{n}\right), P_{\beta_{n}}^{n}\left(x_{n}\right), \cdots, P_{\gamma_{n}}^{n}\left(x_{n}\right)
$$

or a total of $h$ roots. In order that the $\xi_{j}$ may be used to construct a quadrature formula which will exactly integrate the monomials

$$
1, x_{n}, x_{n}{ }^{2}, \cdots, x_{n}{ }^{2 k-1}
$$

they must coincide with the roots of the polynomial $P_{k}^{n}\left(x_{n}\right)$. This is impossible since our assumption that $w_{n}\left(x_{n}\right)$ is non-negative throughout $S^{n}$ assures us that the roots of $P_{k}{ }^{n}\left(x_{n}\right)$ are all distinct and that they separate the roots of $P_{k-1}^{n}\left(x_{n}\right)$ (Szegö [15], p. 45). Thus it is clear that if $h<k$ the $\xi_{j}$ cannot coincide with the roots of $P_{k}^{n}\left(x_{n}\right)$. If $h=k$ it follows that the largest root $\nu_{k}$ of $P_{k}{ }^{n}\left(x_{n}\right)$ has the property

$$
\xi_{i}<\nu_{k}, \quad i=1, \cdots, h
$$

and hence in this case the $\xi_{j}$ also cannot coincide with the roots of $P_{k}{ }^{n}\left(x_{n}\right)$. Thus the solutions of (7.4) cannot serve as points in a quadrature formula of degree $2 k-1$ unless (7.4) coincides with (7.5). This completes the proof.

The solutions of (7.4) may be used to construct integration formulas of degree less than $2 k-1$. However we will not investigate the question of the highest degree formulas which may be constructed from this system. 
8. Systems of Orthogonal Polynomials for Spherical Product Formulas. As shown in Section 3 we can derive spherical product formulas for any integral

$$
\int \cdots_{R} \int w\left(x_{1}, \cdots, x_{n}\right) f\left(x_{1}, \cdots, x_{n}\right) d x
$$

if the region $R$ has the same symmetry as the $n$-dimensional sphere and the weight function (which we assume to be non-negative) is only a function of $r: w\left(x_{1}, \cdots, x_{n}\right) \equiv w\left(x_{1}{ }^{2}+\cdots+x_{n}{ }^{2}\right)$. Thus, the region can be considered as being obtained from a symmetric subset $S$ of the line $-\infty \leqq r \leqq \infty$ by rotating $S$ through all possible directions in the $n$-dimensional space.

Let us denote by $R_{k}{ }^{*}(r) \quad(k=0,1,2, \cdots)$ the sequence of orthogonal polynomials for the integral

$$
\int_{s} w(r) f(r) d r
$$

and, for simplicity of notation, let $Q_{k}{ }^{n}(y)$ denote the $k$-th degree Jacobi polynomial $P_{k}{ }^{[(n-3) / 2,(n-3) / 2]}(y)$. We also define $r_{m}{ }^{2}=x_{1}{ }^{2}+\cdots+x_{m}{ }^{2}(m=1, \cdots, n)$ and $R_{k}\left(r_{n}\right)=R_{k}^{*}\left(r_{n}\right)$ for even $k, R_{k}\left(r_{n}\right)=\frac{x_{1}}{r_{n}} R_{k}^{*}\left(r_{n}\right)$ for odd $k$.

THEOREM. The functions on the left sides of the equations

$$
\begin{aligned}
R_{k}\left(r_{n}\right) & =0 \\
r_{2}{ }^{k} Q_{k}{ }^{2}\left(\frac{x_{2}}{r_{2}}\right) & =0 \\
r_{3}{ }^{k} Q_{k}{ }^{3}\left(\frac{x_{3}}{r_{3}}\right) & =0 \\
\cdots & \\
r_{n}{ }^{k} Q_{k}{ }^{n}\left(\frac{x_{n}}{r_{n}}\right) & =0
\end{aligned}
$$

are all polynomials of degree $k$ in the $n$ variables $x_{1}, \cdots, x_{n}$; each of these polynomials is orthogonal over $R$ with respect to $w\left(x_{1}, \cdots, x_{n}\right)$ to all polynomials of degree $<k$; and the common solutions of this system of equations are the points in the spherical product formula of degree $2 k-1$ for the integral (8.1).

Proof. Each of the polynomials $R_{k}{ }^{*}(r), Q_{k}{ }^{m}(y)$ has the form

$$
\begin{aligned}
& a_{k} x^{k}+a_{k-2} x^{k-2}+\cdots+a_{2} x^{2}+a_{0} \\
& a_{k} x^{k}+a_{k-2} x^{k-2}+\cdots+a_{3} x^{3}+a_{1} x
\end{aligned}
$$

Thus, the functions on the left in (8.2) contain only even powers of $r_{m}(m=2, \cdots, n)$ and are therefore polynomials of degree $k$ in $x_{1}, x_{2}, \cdots, x_{n}$.

Since the distinct values of $\sin \theta_{m-1}=x_{m} / r_{m}$ which determine the points in the spherical product formula satisfy $Q_{k}{ }^{m}(y)=0$ then the points in this formula also must satisfy the system (8.2). There can be no other solutions to this system. The only other way solutions could occur would be if for some $i, r_{i}=0$. If this were true then $x_{1}=\cdots=x_{i}=0$ and we can also see that we must have $x_{i+1}=\cdots=$ $x_{n}=0$. For if $x_{i+1} \neq 0$ then $r_{i+1} \neq 0$ and thus $y=1$ would be a root of $Q_{k}{ }^{i+1}(y)$ 
which is impossible since the roots of this equation all satisfy $-1<y<1$. Hence if $r_{i}=0$ then $x_{1}=\cdots=x_{n}=0$ which can satisfy $R_{k}\left(r_{n}\right)=0$ only when $k$ is odd and this corresponds to one of the original solutions of the system.

Since the spherical product formula is exact for all polynomials of degree $\leqq 2 k-1$ then if $P_{h}\left(x_{1}, \cdots, x_{n}\right)$ is any polynomial of degree $<k$ the formula must be exact for

$$
\int \ldots \int w\left(x_{1}, \cdots, x_{n}\right) R_{k}\left(r_{n}\right) P_{h}\left(x_{1}, \cdots, x_{n}\right) d x
$$

and

$$
\int \cdots_{R} \int w\left(x_{1}, \cdots, x_{n}\right) r_{m}{ }^{k} Q_{k}{ }^{m}\left(\frac{x_{m}}{r_{m}}\right) P_{h}\left(x_{1}, \cdots, x_{n}\right) d x .
$$

Therefore, these integrals are zero and the polynomials in (8.2) are orthogonal to all polynomials of lower degree. This completes the proof.

The authors wish to thank Mrs. Marion Taylor for her assistance in calculating and checking many of the formulas given in this paper.

University of Wisconsin

Madison, Wisconsin

University of Illinois

Urbana, Illinois

1. P. Appell \& J. Kampé de FÉRIet, Fonctions Hypergeometriques et Hyperspheriques; Polynomes d'Hermite, Gauthier-Villars, Paris, 1926.

2. H. S. M. Coxeter, Regular Polytopes, Methuen, London, 1948.

3. V.A. Ditkin, "On certain approximate formulas for the calculation of triple integrals," Dokl. Akad. Nauk SSSR, v. 62, 1948, p. 445-447. (Russian)

4. P. C. Hammer \& A. H. Stroud, "Numerical evaluation of multiple integrals II," $M T A C$, v. 12,1958 , p. 272-280.

5. P. C. Hammer \& A. W. Wymore, "Numerical evaluation of multiple integrals I," $M T A C$, v. 11, 1957, p. 59-67.

6. R. G. Hetherington, Numerical Integration over Hypershells, Thesis, University of Wisconsin, 1961.

7. F. B. Hildebrand, Introduction to Numerical Analysis, McGraw-Hill, New York, 1956.

8. D. JACKSON, "Formal properties of orthogonal polynomials in two variables," Duke Math. J., v. 2, 1936, p. 423-434.

9. V. I. KRYLOv, Approximate Calculation of Integrals, Fizmatgiz, Moscow, 1959. (Russian).

10. W. H. Peirce, "Numerical integration over the planar annulus," J. Soc. Indust. Appl. Math., v. 5, 1957, p. 66-73.

11. W. H. Peirce, "Numerical integration over the spherical shell," $M T A C, \mathrm{v} .11,1957$, p. 244-249.

12. P. Rabinowitz \& G. Weiss, "Tables of weights and abscissas for numerical evaluation of integrals of the form $\int_{0}^{\infty} e^{-x} x^{n} f(x) d x, " M T A C$, v. 13, 1959, p. 285-295.

13. J. RADON, "Zur mechanischen Kubatur," Monatsh. Math. Phys., v. 52, 1948, p. 286-300.

14. A. H. Stroud, "Numerical integration formulas of degree 2," Math. Comp., v. 14, 1960, p. 21-26.

15. G. Szegö, Orthogonal Polynomials, Amer. Math. Soc. Colloq. Publ., v. 23, 1939. 